\title{
Interpretation of VHF ST radar vertical echoes from in situ temperature sheet observations
}

\author{
Hubert Luce and Michel Crochet \\ Laboratoire de Sondages Electromagnétiques de l'Environnement Terrestre, Universite de Toulon et du Var, La \\ Garde, France
}

Francis Dalaudier and Claude Sidi

Service d'Aéronomie du Centre National de la Recherche Scientifique, Verrières Le Buisson, France

\begin{abstract}
The interpretation of the radar aspect sensitivity observed at VHF frequencies in the lower atmosphere is still a subject of controversy in the radar community. Indeed, scattering from anisotropic turbulence layers and partial reflection from stable thin horizontally stratified layers are generally proposed without leading to a definitive conclusion. A cause of this persistent discussion has been a lack of in situ high-resolution observations which could identify and describe accurately the atmospheric structures at the origin of the radar aspect sensitivity. The Radars, Scidar and Balloons (RASCIBA 90) campaign (February-March 1990, Aire sur l'Adour, France) was performed using simultaneously colocated VHF ST radars and balloon experiments. The objective was to obtain information about the smallscale structures of the lower atmosphere and then to identify the origin of radar echoes. The main result was produced by the high vertical resolution $(20 \mathrm{~cm})$ temperature measurements. For the first time, very strong (positive) temperature gradients within thin layers were detected in the lower atmosphere (at least up to $27 \mathrm{~km}$ ). Such an observation allowed then investigation of the partial reflection interpretation using available bifrequency radar measurements: a 45-MHz radar for which typical 15-dB vertical enhancement is observed just above the tropopause and a 72.5-MHz radar for which an aspect sensitivity of the same order is presented for the first time at this frequency. A simple model considering flat and very extended sheets was assumed to estimate quantitatively the possible contribution of these temperature gradients to the VHF radar vertical power. Radar and reconstructed power reflection coefficient profiles are in good agreement in shape and in level showing that partial reflection from atmospheric sheets is an important and generally dominant process at vertical incidence. Furthermore, the effect of sheets of limited extent is investigated using simple theoretical considerations, and this model shows that the correction to the infinite sheet approximation is probably weak. The sheet distortion effect is also approximately evaluated applying Gaussian rough models. The power loss from the flat sheet case at VHF in the vertical direction is weak for a mean roughness height lower than a few tens of centimeters and for larger heights if the correlation length of the irregularities is larger than a few hundreds of meters. Sheet generation mechanisms are also briefly discussed using two previously published models: the viscosity wave model (Hocking et al., 1991) and "sheet and layer" model (Gossard et al., 1985). Neither of the models in their present forms seems to be able to account for the observations.
\end{abstract}

\section{Introduction}

Doppler radars operating in the VHF band have proved to be an efficient method to measure atmospheric

Copyright 1995 by the American Geophysical Union.

Paper number 95RS00713.

0048-6604/95/95RS-00713\$08.00 parameters such as wind velocity and intensity of turbulence especially in the stratosphere and the troposphere. The VHF stratospheric and tropospheric (ST) radar echoes are produced by interaction processes between electromagnetic waves and refractive index fluctuations of the atmosphere. Very soon after their development, it was observed that the echo power depends strongly on the zenithal observation angle and 
is maximum in the vertical direction. This vertical echo enhancement was first noted independently by Gage and Green [1978] at $40 \mathrm{MHz}$ and by Röttger and Liu [1978] at $53.5 \mathrm{MHz}$. Later, many observations were reported, for example, by Sato et al. [1985] at $46.5 \mathrm{MHz}$ and Woodman and Chu [1989] at $52.2 \mathrm{MHz}$. This phenomenon, usually known as "aspect sensitivity," was mainly observed in the lower stratosphere with typical values of enhancements of $10 \mathrm{~dB}$ to $20 \mathrm{~dB}$ above the oblique level and less regularly and less strongly in the troposphere [e.g., Hocking and Röttger, 1983; Tsuda et al., 1988]. Its interpretation is still a subject of controversy in the radar community. Scattering from anisotropic turbulence [e.g., Gage and Balsley, 1980] and partial reflection from thin stable layers [e.g., Röttger and Liu, 1978] are proposed independently or simultaneously to explain this vertical enhancement.

The aim of this paper is to provide some new experimental results in order to contribute to the understanding of this phenomenon. These results were obtained from the 1990 Radars, Scidar and Balloons (RASCIBA 90) campaign using remote sensing and in situ measurements: two VHF ST radars operated by the Laboratoire de Sondages Electromagnétiques de l'Environnement Terrestre (LSEET), a scidar, operated by the University of Nice, and instrumented balloons operated by the Service d'Aerronomie (SA) and the University of Nice. The main information was obtained from the SA high-resolution $(20 \mathrm{~cm})$ vertical profiles of temperature showing the existence at least up to $27 \mathrm{~km}$ of very strong, always positive, temperature gradients within thin layers, the "sheets." These structures were reported and discussed previously in the paper by Dalaudier et al. [1994], hereinafter referred to as DSCV.

In section 2, the characteristics of the VHF radar echoes (dependency in angle, frequency, and time) as well as proposed interpretations of the aspect sensitivity will be reviewed referring to investigations from several authors.

In section 3.1, the aspect sensitivity results (intensity and height variations) for the $45-\mathrm{MHz}$ radar will be outlined, and for the first time, in spite of technological difficulties, some results at $72.5 \mathrm{MHz}$ obtained during RASCIBA 90 will be presented.

Some in situ measurement data, especially highresolution temperature and low-resolution observations, will be presented in section 3.2 referring to DSCV.

In section 4, the partial reflection interpretation will be investigated by estimating the temperature fine structure contribution to the vertical power profile of the backscattered signal. The comparison between observed radar profiles for the two frequencies and reconstructed ones using in situ measurements and a simple model will be shown. The different assumptions and the conditions for which the results are justified will be specified.

Finally, in section 5, possible generation mechanisms of the sheets will be briefly investigated. Important information will be given about the application conditions of the Hocking et al. [1991] theory which introduced viscosity waves as a possible origin of specular reflectors. Gossard et al. [1985] model, associating sheets with turbulent layers will be also discussed.

\section{Generalities of "Aspect Sensitivity"}

\subsection{Echo characteristics}

Green and Gage [1980] hypothesized that the vertical echo power is proportional to the square vertical gradient of potential refractive index $M^{2}$ given by Ottersten [1969a] and therefore to $N^{*}$ (with $N^{2}$ the squared Brunt-Vaisälä angular frequency) if humidity effects are negligible (i.e., in the higher troposphere and in the stratosphere). Experimental investigations confirmed that the parameter $M^{2}$ (or $N^{4}$ in the stratosphere) deduced from in situ measurements (temperature, humidity, and pressure) reproduced the backscattered power received at vertical incidence, indicating a strong correlation between static stability of the medium and the vertical echo power [e.g., Green and Gage, 1980; Gage et al., 1985; Tsuda et al., 1988; Dalaudier et al., 1989, Chu et al., 1990].

Another aspect of the backscattered radar signal is the angular dependence. Due to the difficulty of angular scanning at VHF frequencies, the decrease of the echo power from zenith angles has not been yet clearly measured and results present a large variability both in altitude and in intensity. However, some investigators obtained indications on this subject. For example, Tsuda et al. [1986], using the MU radar (46.5 MHz) pointing in 16 different directions, showed aspect sensitivity up to $10^{\circ}$ from the vertical direction. Hocking et al. [1990, p. 626], using the same instrument, apparently confirmed this observation and reported that "isotropic scatter is almost detected at about 10 degrees". Chu et al. [1990] presented results using the broad beam method introduced by Woodman and Chu [1989] in order to measure aspect sensitivity from Doppler spectra. These authors showed that radar detected isotropic scattering at angles greater than $4^{\circ}$ to $10^{\circ}$, depending on the altitudes.

Papers dealing with radar wavelength dependence showed that aspect sensitivity is not reported at $23 \mathrm{~cm}$ wavelength (corresponding to $1290 \mathrm{MHz}$ frequency) [Balsley and Peterson, 1981]. Other experiments at 430 MHz showed that the isotropic scattering process is also dominant at $70 \mathrm{~cm}$ wavelength [Sato and Woodman, 
1982]. It can be noted that data are not available in the troposphere and the stratosphere at wavelengths larger than $7.5 \mathrm{~m}$ (corresponding to $40 \mathrm{MHz}$ ).

Temporal persistency of some strong echoes observed vertically were reported by Hocking and Röttger [1983]. Using the 150-m range resolution of the Sousy radar, these authors showed echoes persisting at the same height for some tens of minutes and more (longer than $50 \mathrm{~min}$ for one of them). Röttger [1980b] also observed echoes produced by layers located around the tropopause with a lifetime longer than 1 day. Sato et al. [1985] observed with the MU radar layers in the stratosphere with a thickness smaller than the $150 \mathrm{~m}$ radar range resolution. From their Figure 1 or 2 (respectively obtained during winter and summer), a lifetime of several hours can be deduced. A large lifetime can be interpreted as a large horizontal extent since these structures are horizontally advected by the wind field. It is interesting to note that thin layers (about $50 \mathrm{~m}$ thickness) were observed at $430 \mathrm{MHz}$ [Sato and Woodman, 1982] but in this case they were attributed to strong isotropic turbulent layers because aspect sensitivity was not observed.

From these investigations, several mechanisms responsible for the aspect sensitivity have been proposed and are reviewed in the following subsection.

\subsection{Interpretations of Aspect Sensitivity}

Interpretations of aspect sensitivity are still debated since it is difficult to observe the local structure of refractive index fluctuations in three dimensions with sufficient altitude resolution. Furthermore, onedimensional in situ measurements are not sufficient if the fluctuation field is anisotropic.

The aspect sensitivity is clearly due to refractive index structures with much larger coherence length in the horizontal dimension than in the vertical one, but views differ about the nature and the origin of these structures. Two main interpretations are generally given: on one hand, scattering from anisotropic turbulence [e.g., Gage and Balsley, 1980; Doviak and Zrnic', 1984; Waterman et al., 1985, Woodman and Chu, 1989; Dalaudier et al., 1989] and on the other hand, partial reflection from stratified stable horizontally coherent layers [e.g., Gage and Green, 1978; Röttger and Liu, 1978]. The second interpretation assumes the presence of a single or a few horizontal layers in a radar range gate. Fresnel scattering (or coherent scattering) (reflections from many thin layers distributed randomly in the vertical direction) [e.g., Gage et al., 1981; Sheen et al., 1985] and diffuse (or quasi-specular) reflection from thin layers corrugated by turbulence [Röttger, 1980a] were also proposed to explain the overhead enhancement and can constitute extensions of the partial reflection mechanism.

The study of scattering from turbulent media leads to a spectral approach. It was shown [Tatarski, 1961] that the power of the backscattered signal was proportional to the three-dimensional power spectral density of the refractive index fluctuations evaluated at half the radar wavelength for monostatic radars. The turbulent eddies would become anisotropic (i.e., their statistical properties depend on direction) at larger scales in the "buoyancy subrange" separated from the isotropic turbulence by the transition scale $L_{b}$ [e.g., Shur, 1962; Lumley, 1964; Weinstock, 1978; Hocking, 1985]. Anisotropy models were presented by Doviak and Zrnic' [1984], Waterman et al. [1985], Dalaudier et al. [1989], and Gurvich and Kon [1993].

Nevertheless, the anisotropic turbulence interpretation was not accepted by all the radar community. For instance, Gage et al. [1981] argued that this interpretation does not seem compatible with highstability conditions of the atmosphere which are observed with strong vertical echoes. Röttger [1980a] showed that the temporal correlation of the vertical signals were too large to be attributed to typical turbulent echoes.

The partial (or Fresnel) reflection theory assumes the existence of steplike structures or sharp refractive index gradients from thin layers with large horizontal extent (i.e., greater than the Fresnel length). Due to horizontal stratification, partial reflection would be preponderant in vertical direction and would explain the strongest echoes in this direction relative to oblique ones. Very early this mechanism was put forward to interpret transhorizon bistatic links (at grazing angles) in the lower troposphere by du Castel et al. [1966] and Saxton et al. [1964] and was supported by in situ measurements of refractive index sheets using microwave refractometers. From different analyses of these measurements Misme et al. [1958] proposed the presence of atmospheric sheets (at least, in the first kilometers of altitude) with horizontal dimensions of several kilometers and thickness of several meters.

Scattering from isotropic turbulence, as developed first by Booker and Gordon [1950] and improved by Tatarski [1961] and Ottersten [1969b], is generally accepted to explain the echo power at oblique incidence (with zenithal angle greater than $10^{\circ}$ according to the various observations already mentioned) and is combined with the partial reflection mechanisms at and near the vertical direction.

However, Woodman and Chu [1989] argued that the partial reflection mechanism must be rather exceptional at VHF frequencies because very thin layers of the order of a few meters are needed. They claimed that such 
structures are "impossible to accept" because temperature increases (of the order of $0.5^{\circ}$ ) can't be reached without being dynamically unstable and then without being destroyed by turbulent diffusion before their complete formation. They claimed also that angular dependence around the vertical direction would be sharp which is not observed by experiments, especially by their broad beam method. In fact, while anisotropic turbulence seems to be more appropriate to interpret the observed angular dependence (although the agreement is strongly a function of the model), diffuse reflection occurring from a rough surface (applied by Ratcliffe [1956] to the ionosphere and reintroduced in atmosphere studies by Röttger [1980a]) can also reduce the specular nature of the partial reflection by broadening the scattered beam. This interpretation can be considered to be consistent with Misme et al.'s [1958] or du Castel's [1966] sheet distortion observations in the lower troposphere.

The wavelength dependency predicted by both kinds of interpretation is strongly dependent on the assumed model. Dalaudier et al. [1989] investigated an anisotropic model of temperature fluctuations based on balloon observations. It predicted a $\lambda^{3}$ dependency of the three-dimensional spectra corresponding to a -3 slope of the scalar spectrum. The wavelength dependency predicted by models based on "partial reflection" is strongly dependent on the shape of the reflecting structures assumed by the models [e.g., Green et al., 1979; Woodman and Chu, 1989]. As a general rule, the smoother structure leads to the stronger wavelength dependency.

Although these two main mechanisms, scattering from anisotropic turbulence and partial reflection from stable layers, are usually introduced independently, Doviak and Zrnic' [1984] showed that it is possible to unify these two concepts. From the Born approximation of the scattered field and including the effect of the curvature of the wave front by expanding the phase term at the second order, they obtained a general formula involving the three-dimensional (3-D) (anisotropic) spectrum. They showed that this formula is identical to the "partial reflection" one in the case of an infinite horizontal anisotropy. On another hand, some investigators have proposed a combination of these two mechanisms. For example, Hocking et al. [1990] suggested a combination of anisotropic turbulence confined at the edges of turbulent layers (also proposed by Woodman and Chu [1989]) and specular reflectors. The proposition of Hocking et al. [1991] for viscosity and thermal conduction waves as the origin of such reflectors will be discussed in section 5 .

\section{Experimental Results}

The RASCIBA experiment took place at Aire sur $1^{\prime}$ Adour $\left(0^{\circ} 15^{\prime} \mathrm{W}, 43^{\circ} 42^{\prime} \mathrm{N}\right)$ in February and March 1990. Three kinds of instrumentation were used in order to study the fine scale structures of the lower atmosphere and to compare measurements from Radars, Scidar and Balloons. The radars used were the 45-MHz ST VHF radar "Provence" and the "INSU Meteo" 72.5-MHz ST VHF radar operated by the LSEET. Their main characteristics are given in Figure 1. The scidar was operated by the University of Nice but its results will not be presented in this paper. Four balloon flights were performed during four nights and one or two gondolas were carried by each balloon. A brief description of their instrumentation is also given in Figure 1 and more precisely in DSCV.

\subsection{Radar Measurements}

Figure 2 displays 45-MHz oblique and vertical signal to noise ratio $(S / N)$ profiles averaged on about 1 hour during the four balloon flights. They were obtained from $2250 \mathrm{~m}$ up to $18,450 \mathrm{~m}$ with a 600 -m sampling interval and a 600-m resolution (4- $\mu$ s pulse duration). A typical vertical enhancement appears always around $11.5-12 \mathrm{~km}$ (about $15 \mathrm{~dB}$ ). The first vertical echo profile (obtained on February 19) is particularly stronger than the oblique one beyond $8 \mathrm{~km}$ and below $4.5 \mathrm{~km}$. A more isotropic zone (which corresponds to an oblique enhancement) is observed between these two heights. The second $S / N$ profile (from February 20) shows the weakest aspect sensitivity below $8 \mathrm{~km}$ (i.e., in the troposphere) and above $14 \mathrm{~km}$ (less than $5 \mathrm{~dB}$ ). The third one (on February 22) reveals aspect sensitivity for all altitudes but with decreasing intensity below $6 \mathrm{~km}$. The last day of observation (on March 1) displays the strongest aspect sensitivity (mainly in the troposphere) with a $30-\mathrm{dB}$ maximum at $6450 \mathrm{~m}$.

Finally, these observations show that the vertical echoes are always stronger than the oblique ones just above the tropopause identified by the balloon (marked by arrows) and in the lower stratosphere, but the aspect sensitivity is intermittent in time in the troposphere. The first three periods correspond to weak wind conditions and the fourth one to disturbed meteorological conditions (see rawinsonde measurements obtained at Bordeaux $\left(0^{\circ} 35^{\prime} \mathrm{W}, 44^{\circ} 50^{\prime} \mathrm{N}, 128 \mathrm{~km}\right.$ northward from Aire sur l'Adour), Figure 2 of DSCV).

Because of technical difficulties (like Doppler artifacts and coding problems), the data sets were difficult to analyse at $72.5 \mathrm{MHz}$ and only one period without coding is presented in Figure 3. S/N ratio profiles were obtained with a 750-m sampling interval and a 2400-m 


\begin{tabular}{||l|l|l||}
\hline \hline Parameters & Provence & Insu Meteo \\
\hline \hline Frequency & $45 \mathrm{MHz}$ & \\
\hline Antenna type & colinear coaxlal & colinear coaxial \\
\hline Antenna dmension & $\# 2500 \mathrm{~m}^{2}$ & $\# 1290 \mathrm{~m}^{2}$ \\
\hline Pulse peak power & $\# 18 \mathrm{KW}$ & $\# 4.2 \mathrm{KW}$ \\
\hline Pulse width & $4 \mathrm{\mu s}$ & $16 \mu^{\mathrm{s}}$ \\
\hline Height resolution & $600 \mathrm{~m}$ & $2400 \mathrm{~m}$ \\
\hline Sampling interval & $600 \mathrm{~m}$ & $750 \mathrm{~m}$ \\
\hline Tme resolution & $6 \mathrm{mn} 30 \mathrm{~s}$ & $1 \mathrm{mn} 45 \mathrm{~s}$ \\
\hline Beam width & $\# 5^{\circ}$ & $\# 5^{\circ}$ \\
\hline Beam pointing direction & Vertical & Vertical \\
\hline & 2 obliques : & 2 obllques : \\
\hline & Azimuts: $225^{\circ} / 135^{\circ}$ & Azimuts: $0^{\circ} / 90^{\circ}$ \\
\hline Temp. cosmic nolse & $\# 6000 \mathrm{~K}$ & $\# 2200 \mathrm{~K}$ \\
\hline Receiver noise & $\# 300 \mathrm{~K}$ & $\# 300 \mathrm{~K}$ \\
\hline \hline
\end{tabular}

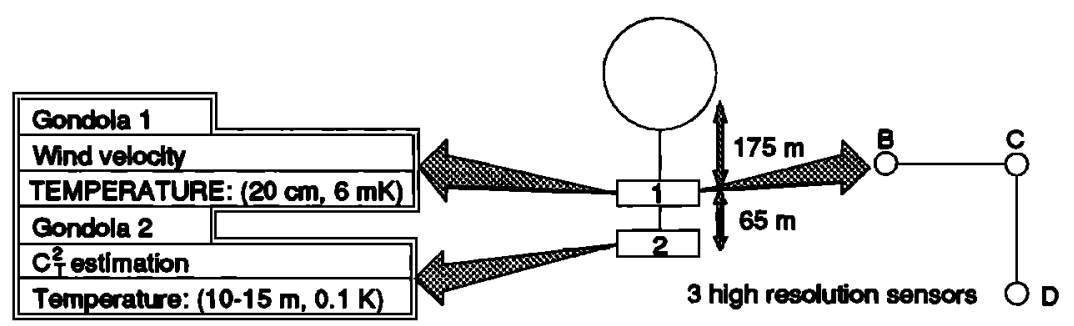

Figure 1. System parameters for Provence and Insu Meteo ST radars and gondola description.

range resolution (16- $\mu$ s pulse duration) and averaged over about $4 \mathrm{~min}$. Vertical enhancement of about $15 \mathrm{~dB}$ is also observed at this frequency around $11 \mathrm{~km}$. The vertical $\mathbf{S} / \mathbf{N}$ ratio seems also slightly stronger than the oblique in the troposphere. The enhanced oblique (dotted line) $\mathrm{S} / \mathrm{N}$ around $14 \mathrm{~km}$ could be due to a residual Doppler artifact and may not have geophysical origin. However, it is interesting to note that the aspect sensitivity is still large at a radar wavelength of $4 \mathrm{~m}$.

\subsection{In Situ Measurements}

The description of the in situ measurements will be limited to the minimum required for the estimation of the reflection factor. The reader should refer to DSCV for more details.

During the continuous radar measurements, four instrumented balloons were launched at the same site and during the night in order to avoid the effects of insolation on the sensors. Each balloon carried two gondolas (or one for the first flight). The main one, hung $175 \mathrm{~m}$ below the balloon itself, was operated by the Service d'Aéronomie and gave high-resolution (better than $20 \mathrm{~cm}$ ) vertical temperature measurements owing to three fast response sensors (with time constant less than $10 \mathrm{~ms}$ at all altitudes and placed $1 \mathrm{~m}$ apart at the corner of a square). This gondola also carried a high- resolution pressure sensor, allowing an accurate determination of the gondola altitude by integration of the hydrostatic equation. The second gondola, hung $65 \mathrm{~m}$ below the first, was operated by the Astrophysic Department of the University of Nice and was intended for coarse $C_{T}^{2}$, temperature, and humidity measurements.

Temperature measurements. The high vertical temperature resolution allowed the detection of very thin layers (typically less than $10 \mathrm{~m}$ ) with strong positive gradients from about 30 to $300 \mathrm{~K} \mathrm{~km}^{-1}$. These kinds of structures have already been observed in the 1960s in the lower troposphere [Misme et al., 1958; Saxton et al., 1964] using refractometers, and more recently, Gossard et al. [1985] described such structures for temperature, humidity, and wind using the 300-m meteorological Boulder Atmospheric Observatory (BAO) tower. However, to our knowledge, the DSCV paper constitutes the first report of thin temperature sheets at least up to $27 \mathrm{~km}$. It is also interesting to note that similar structures were observed in the ocean [e.g., Luek, 1988] or in lakes [e.g., Imberger and Ivey, 1991].

An example of these sheets is given in Figure 4 and additional examples are given in DSCV. This Figure shows three successive close-ups (2000, 200 and $20 \mathrm{~m}$ ) from the complete profile (see Figure 6) obtained from the ground up to $27 \mathrm{~km}$ for the fourth flight. In the third 


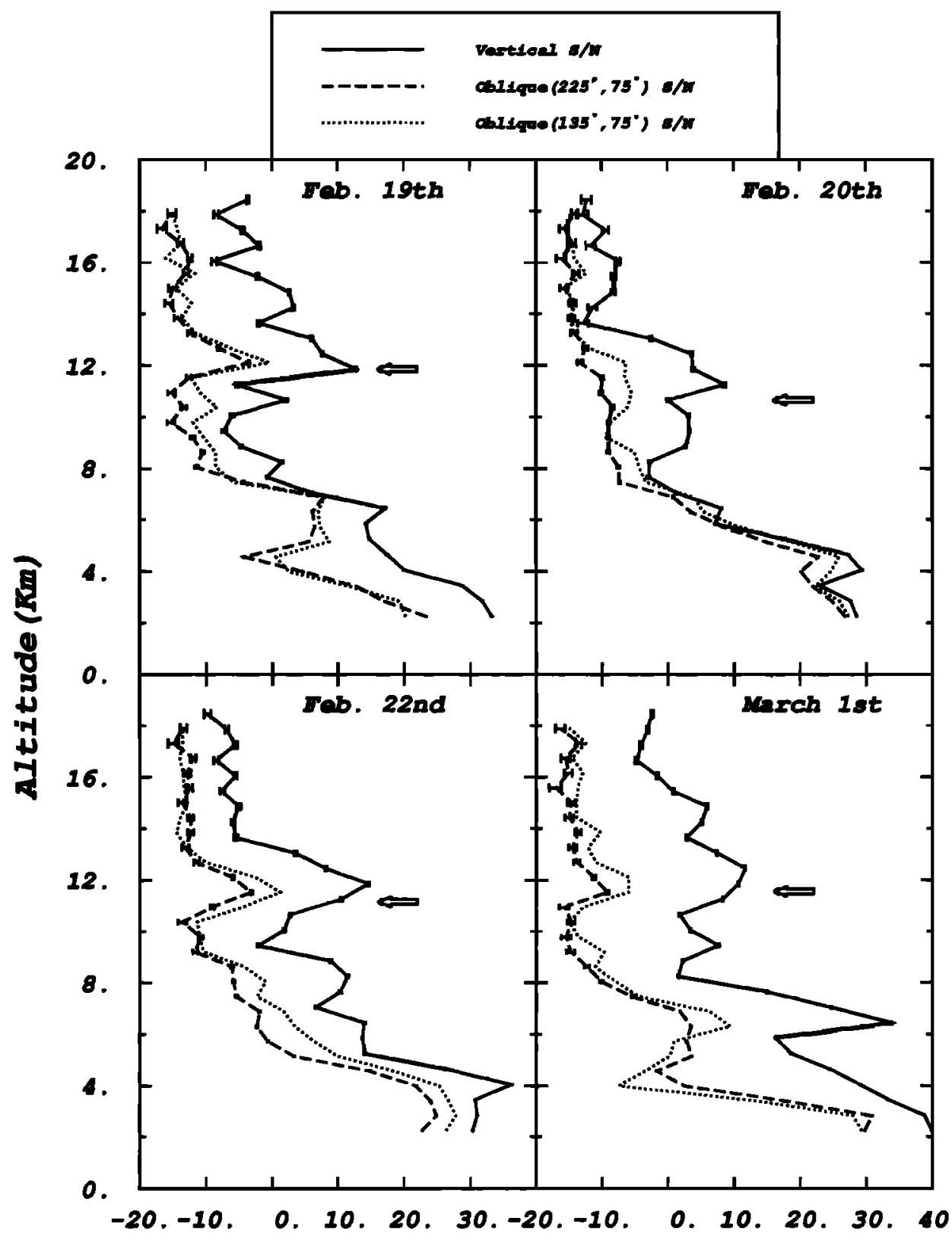

\section{$S / N(d B)$}

Figure 2. The 45-MHz oblique and vertical signal to noise ratio profiles averaged on about 1 hour during the four balloon ascents. The strongest aspect sensitivity zone appears just above the tropopause. The most disturbed meteorological condition day (March 1) shows the strongest aspect sensitivity, while February 20 (corresponding to weak wind conditions) shows the weakest one in the troposphere and beyond $14 \mathrm{~km}$. The small arrows indicate the tropopause level determined from the temperature soundings and by using the meteorological criterion. Small horizontal bars show the estimation errors from the moment method for the vertical and the first oblique profiles (for more legibility, the second oblique profile errors are not presented). 


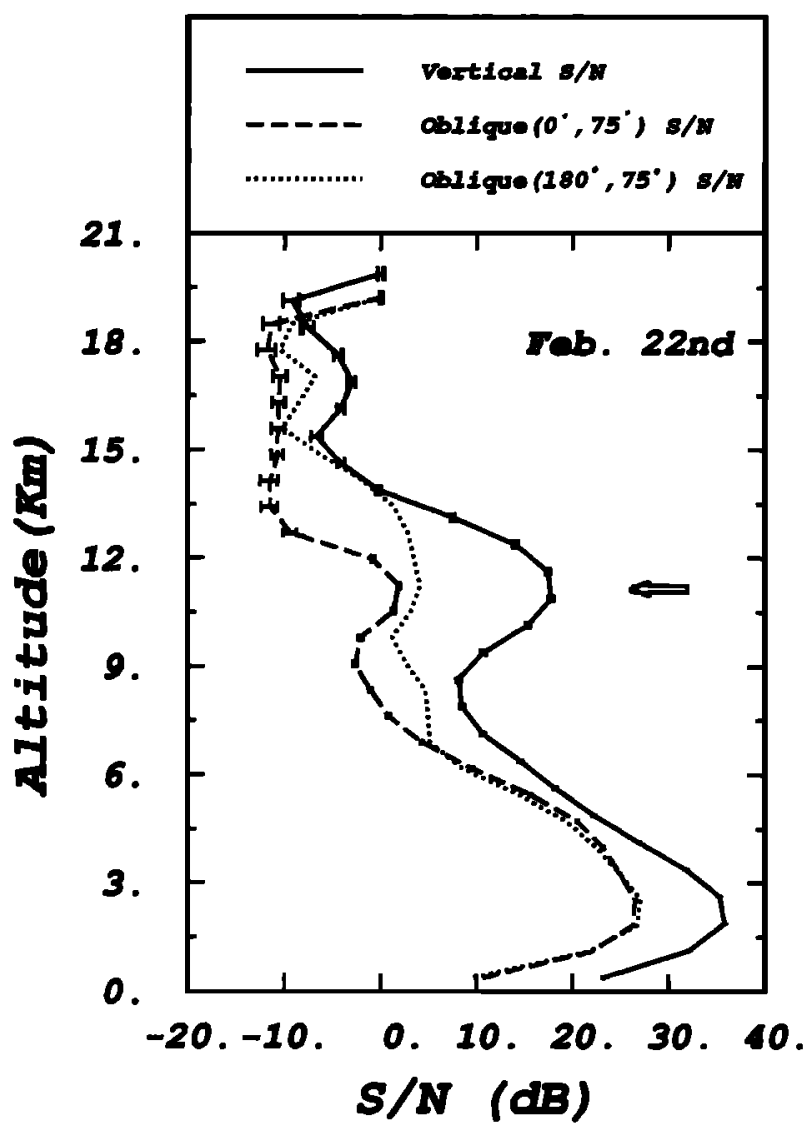

Figure 3. The 72.5-MHz oblique and vertical signal to noise ratio profiles averaged on about 4 min during the third balloon flight. The oblique $\left(180^{\circ}, 75^{\circ}\right) \mathrm{S} / \mathrm{N}$ enhancement with respect to the oblique $\left(0^{\circ}, 75^{\circ}\right) S / N$ profile can be due to a residual Doppler artifact and may not have a geophysical sense. Strong aspect sensitivity can be noted around $11-12 \mathrm{~km}$.The small arrows indicate the tropopause level determined from the temperature soundings and by using the meteorological criterion. Small horizontal bars show the estimation errors from the moment method for the vertical and the first oblique profiles (for more legibility, the second oblique profile enrors are not presented).

frame all three sensor measurements are shown. The sheet has a thickness of $3.6 \mathrm{~m}$ and corresponds to a temperature increase of $0.58 \mathrm{~K}$, or a gradient of $160 \mathrm{~K}$ $\mathbf{k m}^{-1}$.

The notion of a sheet is somewhat arbitrary and cannot be connected only to the gradient threshold. The procedure, described in DSCV, selects temperature gradients that were shown to be generally compatible with the expected properties of sheets (i.e., large horizontal extent, strong temperature increase and thinness). This procedure consists of the following steps. The potential temperature profile $\Theta(z)$ is low-pass filtered with a cutoff of $10.8 \mathrm{~m}$. An $N^{2}$ (squared Brunt-Vaïsälä frequency) profile is computed at $5.4 \mathrm{~m}$ (Nyquist) resolution. Then, $N^{2}$ values larger than a given threshold $\left(10^{-3}\right.$ in the troposphere and $2.10^{-3}$ in the stratosphere) are selected. Finally, after rejecting spurious regions (usually due to turbulent wake of the balloon), the thickness $\Delta z$ was determined manually and the temperature increase $\Delta T$ was deduced accordingly using the high-resolution profile. The parameters of this procedure are quite arbitrary. However, the cut-off frequency of the filtering was chosen in order to suppress (as far as possible) the random fluctuations and instrumental noise while keeping a good vertical resolution. The thresholds were chosen in order to select the strongest sheets (the main objective of the DSCV paper). A sensitivity analysis shows that the main parameter which contributes to the variation of the number of selected sheets is the threshold. In the present work another threshold will be introduced in order to test the influence of this parameter on the reconstructed profiles presented in section 4 . The main conclusions of the sheet analysis are presented here. More details are given in DSCV and the reader is invited to refer to it. Most of the sheets appear above the tropopause, but some can be observed in the troposphere (Figure 6). They are frequently observed in groups and seem to be connected with meteorological conditions (with the largest occurrence during the fourth flight corresponding to disturbed conditions, with wind speed larger than $40 \mathrm{~ms}^{-1}$ between 5 and $10 \mathrm{~km}$ ). It could be noted that some of them are thinner than $2 \mathrm{~m}$ and reach a temperature gradient greater than $200 \mathrm{~K} \mathrm{~km}^{-1}$.

Information about horizontal extent is difficult to obtain from these in situ data sets because of the lack of high-resolution measurements in the horizontal direction. However, a preliminary conclusion was deduced in DSCV from comparisons between high-resolution sensor profiles of the main gondola and coarse resolution sensor (about 10-m and 0.1-K resolutions) profiles of the second gondola, $65 \mathrm{~m}$ below the main one. It was concluded in DSCV that their typical horizontal extent is possibly much larger than $50-100 \mathrm{~m}$ and their lifetime was much longer than $12 \mathrm{~s}$ for the most important sheets. Sheet roughness was also noted from these two measurements (Figure 4 of DSCV) but the different spatial resolution of the two sensors lead to uncertainties about the magnitude of this distortion. The extreme estimations of the distortion were made for each sheet observed from two points of measurement horizontally separated. These estimations define a confidence interval in which the "true" distortion should be found. Consequently, a realistic estimate of the root mean square (rms) vertical distortion should thus be significantly smaller than the 


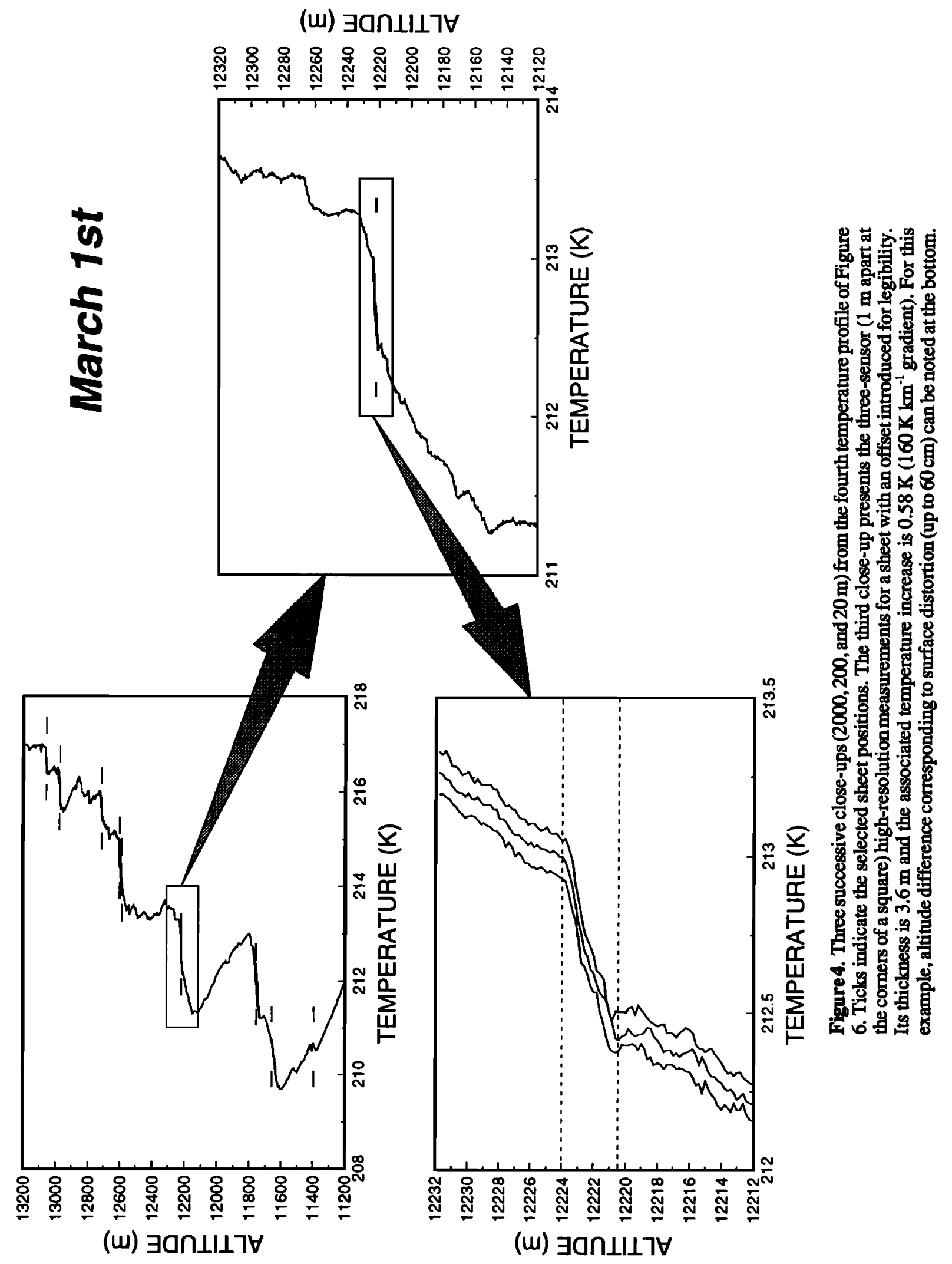




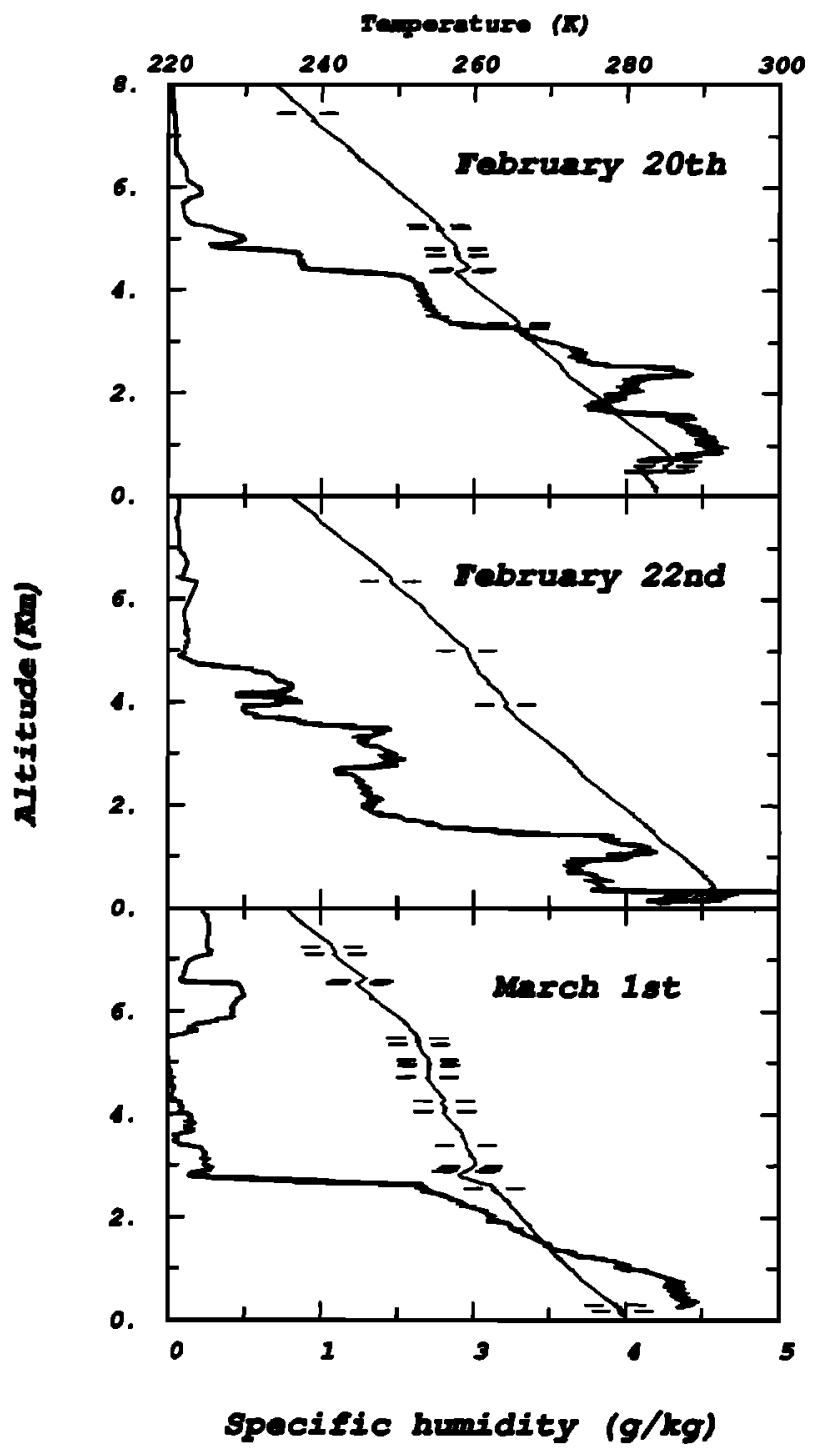

Figure 5. Specific humidity data (grams per kilogram) obtained by the second gondola (thick solid line) between 0 and $8 \mathrm{~km}$. The high-resolution temperature profile (thin solid line) is superimposed with selected sheet positions from the DSCV criterion using the lower threshold (thin horizontal ticks) and the higher one (thick horizontal ticks). Note the correlation between sheet position and the humidity gradients.

raw Ims estimation ( $5.9 \mathrm{~m}$ ) from DSCV data. Using the available observations, we can temporarily conclude that the vertical distortion is larger than a few tens of centimeters (the sheets are definitely rough) and smaller than a few meters. Future measurements should improve this coarse estimation.
It is interesting to note that Misme et al. [1958] and du Castel [1966] studied the same kind of stable sheets of refractive index in the lower troposphere and gave details about their mean characteristics from their observations obtained from refractometers carried by aircraft. Du Castel proposed that sheets are distorted by primary and secondary irregularities. The primary ones would have a characteristic height of several meters with some tens of meters length, and the secondary ones, characteristic height of some tens of meters with several kilometers length. But it was specified that these orders of magnitude were deduced from few measurements and therefore must be considered with caution.

Humidity measurements. Humidity usually plays an important role in the echo power generation in the lower troposphere and should be taken into account below $8 \mathrm{~km}$ [e.g., Gage and Balsley, 1980]. Because of the poor altitude resolution, it is impossible to identify humidity sheets. However, the humidity data (Figure 5) show the presence of strong gradients, some of them being correlated with temperature sheets; see, for example, around $3.5 \mathrm{~km}$ and $4.5 \mathrm{~km}$ on February 20 or around $6.5 \mathrm{~km}$ and $7.1 \mathrm{~km}$ on March 1 . Note that these humidity gradients are not necessarily negative. Others are not associated with temperature sheets, for example, at $2.4 \mathrm{~km}$ on February 20 or at $1.5 \mathrm{~km}$ on February 22 . Consequently, the presented data do not allow to confirm or to infirm a very high correlation between humidity and temperature sheets in the planetary boundary layer as reported by Gossard et al. [1985].

\section{Sheet Contribution to Radar Echoes}

In light of these new observations it is interesting to investigate the possible sheet contribution to the vertical echo enhancement. Our first approach is essentially qualitative. The simultaneity of in situ and remote sensing measurements performed around the same location allows a direct comparison of the two kinds of data sets. A quite good correlation is observed between vertical radar echo enhancement and temperature sheet positions. For instance, it is striking to note that sheets identified around the tropopause correspond very well to vertical echo enhancements (Figure 6). When the temperature is not changing abruptly at the tropopause, as on February 20, the presence of sheets on each side of the tropopause and the vertical echo enhancements at these altitudes varies accordingly.

The next approach is quantitative, and its objective is to determine power reflection coefficient profiles from the high-resolution temperature data using a simple model and to compare them to the radar echo power profiles expressed in term of power reflection coefficient. 


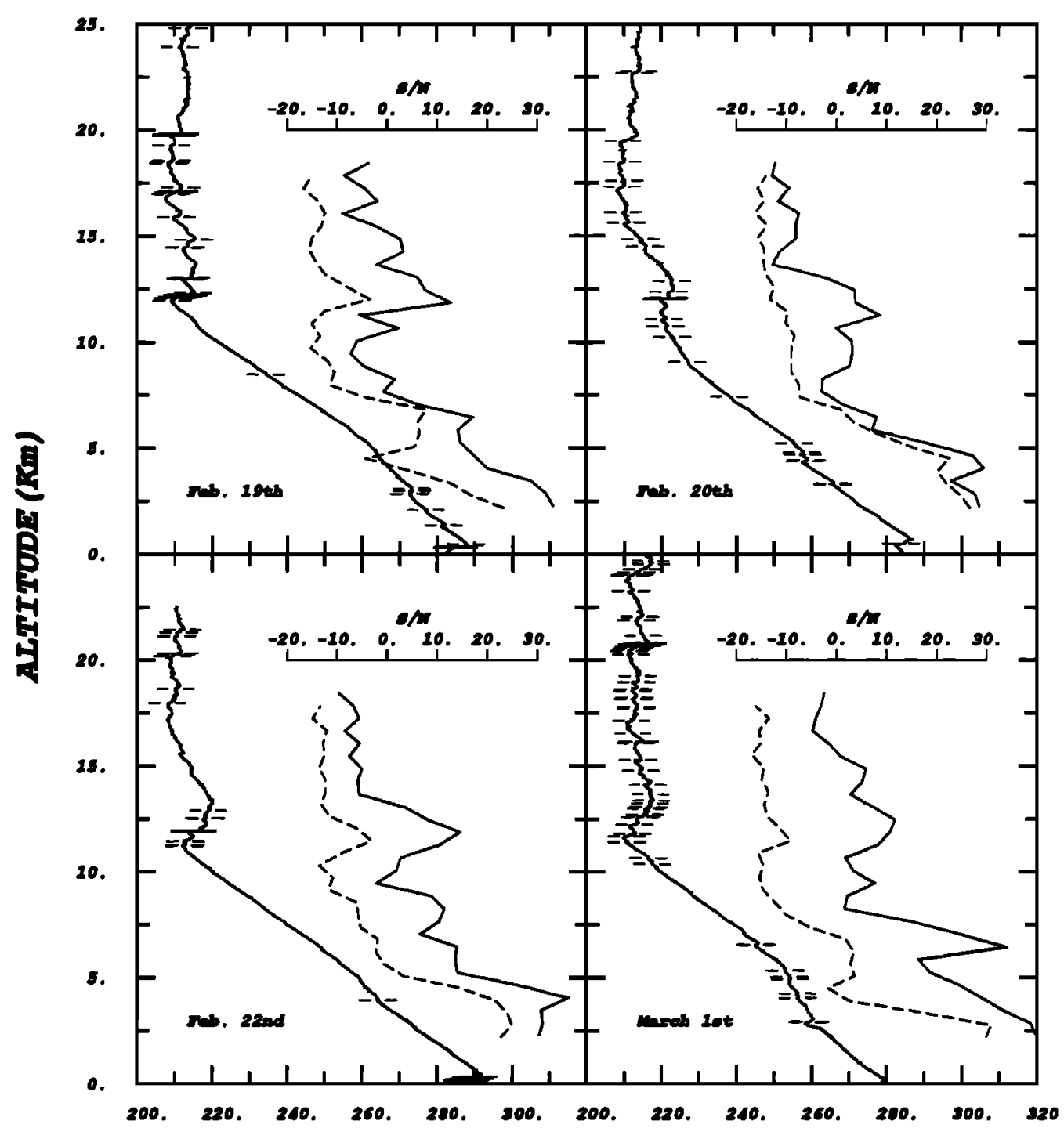

\section{TRAMPERATURE (K)}

Figure 6. High-resolution temperature profiles up to $25 \mathrm{~km}$ for the four balloon ascents. Small ticks show the sheet positions selected from the DSCV criterion. They mainly occur just above the tropopause and in the stratosphere (in regions of high static stability) but can be also observed in the troposphere. The most disturbed day (March 1) indicates the largest number of sheets, especially in the stratosphere. At the right of each temperature profile, the vertical and oblique (1) $\mathrm{S} / \mathrm{N}$ is presented to show the good correlation between vertical radar echoenhancement and temperature sheet positions particularly around the tropopause. The strong enhancement observed on March 1 is associated with simultaneous temperature sheets and strong humidity gradient (Figure 5).

\subsection{Hypotheses and Approximations}

1. Sheet surfaces are assumed to be flat (distortions effects are not taken into account but will be discussed in section 4.5).
2. Due to various effects (such as sheet distortions, horizontal and vertical advections), vertical separation between sheets may be varying randomly. Consequently, interferences between sheets in the same radar volume may be also varying randomly. In these 
conditions the received average power is obtained by summation of the backscattered powers from each sheet.

3. In order to use a simple reflection computation model, the horizontal dimension of the sheets is assumed to extend beyond the first Fresnel zone of radius $R_{f}=\sqrt{r \lambda / 2}$ (see Table 1 for an evaluation) independently of the sheet thickness. See discussion in section 4.4 for limited extent correction.

4. Temperature sheets measured by the balloon are supposed to be representative of temperature sheets in the radar volume of resolution. In other words, the horizontal extent of the regions favorable to sheet existence for the specific radar resolution must be thus greater than several tens of kilometers.

\subsection{Computation Method of the Power Reflection Coefficient Profiles From Selected Sheets}

The propagation of an incident electromagnetic wave in a stratified medium leads to a partially reflected wave. The reflection coefficient $p$ is the ratio between the complex amplitude of the incident and reflected plane waves. The power reflection coefficient from a horizontally stratified layer is obtained from the generalized WKB solution and by neglecting the multiple reflections within the layer. Its expression is given by Wait [1970]:

$$
|\rho|_{\text {cheat }}^{2}=\frac{1}{4}\left|\int_{\frac{L}{2}}^{\frac{L}{2}} \frac{1}{n} \frac{d n}{d z} \exp \left(-i k_{b} z\right) d z\right|^{2}
$$

where $L$ is the thickness of the layer, $k_{b}$ the Bragg wavenumber such as $\lambda_{b}=\lambda_{\text {radd }} / 2$, and $n(z)$ the refractive index profile in the layer. This result can be applied only if the horizontal dimension of the stratified layer extends beyond the first Fresnel zone. For example, the layer must cover at least a $400-\mathrm{m}$ diameter at $12 \mathrm{~km}$ range for $45 \mathrm{MHz}$ frequency.

Unfortunately, humidity data sets are not available with a sufficient resolution to observe humidity sheets. This is why computations are made with only the dry refractive index profile, valid wherever humidity gradients are negligible. Neglecting humidity, (1) becomes approximately

$$
|\mathrm{P}|_{\text {dheow }}^{2}=\frac{1}{4}\left(\frac{77.6 x 10^{-6} P_{m}}{T_{m}^{2}}\right)^{2} \cdot\left|\int_{\frac{L}{2}}^{\frac{L}{2}} \frac{d T}{d z} \exp \left(-i k_{b} z\right) d z\right|^{2}
$$

where $P_{\mathrm{m}}$ and $T_{\mathrm{m}}$ are, respectively, the mean pressure (in mbars) and the mean temperature (in Kelvin) in the sheet, and $T(z)$ the temperature profile (in Kelvin) of the sheet.
For each selected sheet, we computed numerically the power reflection coefficient by using the relation (2). The $20-\mathrm{cm}$ resolution of the balloon measurements is adequate for a numerical computation of the reflection coefficient. In order to reduce the small scales fluctuations (due to possible turbulence within the sheets), the three sensor profiles have been averaged, contrary to DSCV work for which only one of the three sensors was used.

For each radar range gate characterized by index $i$, the estimated contribution of the sheets $|p|_{i \text { in }}^{2}$ is computed as a weighted average of the power reflection coefficient of each sheet:

$$
|p|_{i_{\text {radur }}^{2}}^{2}=\sum_{j=1}^{j=N_{i}} W^{2}\left(z_{j}\right)|p|_{j, i_{\text {dheo }}}^{2}
$$

where $N_{\mathrm{i}}$ is the number of selected sheets in the corresponding gate. The amplitude weighting function $W(z)$ is a convolution between the transmitted radar pulse and the impulse response of the matched filter of the receiver. $W^{2}(z)$ is approximated by a symmetric triangle with a full width at half maximum equal to the radar range resolution ( $600 \mathrm{~m}$ for $45 \mathrm{MHz}$ and $2400 \mathrm{~m}$ for $72.5 \mathrm{MHz}$ ). This computation method was thus applied to the sheets selected by the DSCV procedure. But the choice of the threshold parameter is not necessarily appropriate to our present computation (as already explained in a previous section), and some sheets, even less strong, can account for part of the power reflection coefficient. The sheets selected in DSCV will be compared here with results from another set of sheets deduced from different thresholds. This set was obtained by decreasing the thresholds to $1.5 \times 10^{-3}(\mathrm{rad} / \mathrm{s})^{2}$ in the stratosphere instead of $2 \times 10^{-3}$ and to $7.5 \times 10^{-4}(\mathrm{rad} / \mathrm{s})^{2}$ in the troposphere instead of $10^{-3}$ (corresponding to a decrease of 25\%). The main consequences are an increase of sheet number by $72 \%$ (from 189 to 325 ) and the decrease of their mean temperature increment by $35 \%$ (from $0.4^{\circ}$ to $0.26^{\circ}$ ).

We deduced the radar power reflection coefficient profile by the relation (e.g., from Gage and Green [1978])

$$
|\rho|_{\text {radur }}^{2}=\frac{8 k T_{N} c \lambda^{2} r^{2}}{\alpha P_{r} A_{e}^{2} N_{c} \Delta r} \frac{S}{N}
$$

$\mathrm{S} / \mathrm{N}$ is the signal to noise ratio, $P_{t}$ the transmitted power, $A_{\text {o }}$ the effective area of the antenna, $\alpha$ the transmission line and antenna efficiency, $r$ the range, $N_{c}$ the number of coherent integrations, $\Delta r$ the range resolution, $c$ the light celerity, $k$ Boltzmann's constant, and $T_{\mathrm{N}}$ the cosmic noise temperature. 


\subsection{Results of the Comparisons}

Figures 7 display the comparison between the observed vertical radar power reflection coefficient profiles computed with (4) and the reconstructed ones computed with (2) and (3) from the two different sets of selected sheets.

Several radar profiles obtained during the balloon ascent are shown. The results are given only for the third flight at $72.5 \mathrm{MHz}$. The interruption of the reconstructed profiles means a local absence of sheets according to the selection procedure. We note first that the observed and calculated profiles fit quite well in shape. Enhancements at the tropopause level are identified by both radars and theoretical calculations and extrema locations of reconstructed profiles are in good agreement with the $45-\mathrm{MHz}$ and $72.5-\mathrm{MHz}$ radar observations for the two sets. The power reflection coefficient level is also well reproduced without amplitude normalization. This result is interesting if we assume that the radars were well calibrated in power level. However, some uncertainties still exist about the estimated radiated power, antenna loss or temperature noise. The present calibration is in agreement with previous comparisons with scidar measurements [Vernin et al., 1990] and with simultaneous oblique comparisons with scidar (Luce et al., paper in preparation, 1995). An error of calibration at $45 \mathrm{MHz}$ could produce a change of the power reflection coefficient of several decibels but wouldn't be prejudicial to our main conclusion: the comparison leads to the same order of magnitude in power level. As concerning the $72.5-\mathrm{MHz}$ radar calibration, the uncertainties have not yet been clearly established and comparisons in power level must be made with caution.

The comparison between power reflection coefficient profiles obtained from the two sets of sheets shows that the lowest threshold choice extends and enhances the reconstructed profiles. This is especially the case on February 19 at $6.5 \mathrm{~km}$ and $10.5 \mathrm{~km}$ and on February 22 at $6,8.5$, and $16 \mathrm{~km}$. The lowest threshold implies also enhancements of the computed profiles for example on February 19 around $16 \mathrm{~km}$ and on February 20 around $9 \mathrm{~km}$. However, the general conclusions are the same for both sheet selection procedures. This means that the reconstructed power reflection coefficient profiles are not qualitatively very sensitive to the threshold. This result can be understood in the following way: a lower threshold leads to the selection of a larger number of sheets, but with a much smaller individual contribution to the power reflection coefficient (2) and probably with a smaller horizontal extent. Furthermore, this smaller threshold also introduce temperature fluctuations resulting presumably from turbulence, which are not in agreement with the model used. However, in some altitude range, the difference between the two reconstructed profiles can be significant if the number of additional small sheets is locally large. The contribution of the turbulent structures to the radar signal should be accounted for with a different kind of model.

Some discrepancies between radar and reconstructed profiles can still be noticed:

1. Vertical enhancements between 7 and $9 \mathrm{~km}$ on February 22 and between 8 and $10 \mathrm{~km}$ on March 1 are not (or poorly) explained by the presence of sheets. The power reflection coefficient level on March 1 is strongly underestimated in the troposphere. In fact, humidity sheets were observed by Gossard et al. [1985] in the boundary layer, and it is reasonable to think that it is likewise true in all zones where humidity is present, as suggested by our humidity measurements (see Figure 5). The reflection coefficient computed from the dry refractive index would then be obviously underestimated in the case of the presence of humidity sheets although a confirmation is necessary.

2. Strong computed reflection coefficient at 14,850 $m$ on February 20 corresponds to the presence of very thin sheet of about 1-m thickness with strong temperature increase $(0.27 \mathrm{~K})$. Such a strong gradient $(\approx 300 \mathrm{~K}$ $\left.\mathbf{k m}^{-1}\right)$ is exceptional and it is not surprising that it is not observed in the radar lobe at the same altitude. Furthermore, because this gradient is so thin, we expect that its horizontal extent is limited and possibly smaller than the Fresnel scale, thus leading to a further reduction of the reconstructed reflected power.

\subsection{Limited Horizontal Extent Effects}

If the horizontal dimension of a single stratified layer extends beyond the first Fresnel zone, the received power $P_{\mathrm{r}}$ at vertical incidence from a flat and horizontal reflecting layer at $r$ range is given by the basic relation [Friend, 1949]

$$
P_{r}=\frac{\alpha^{2} P_{t} A_{a}^{2}}{4 \lambda^{2} r^{2}}|\rho|_{\text {theoct }}^{2}=K|\rho|_{\text {abeot }}^{2}
$$

$P_{\mathrm{t}}$ is the transmitted power, $A_{\mathrm{e}}$ the effective area of the antenna and $\alpha$ the transmission line and antenna efficiency. $K$ is a function of $r$, distance sheet radar, and will be used in the power evaluations in the following sections.

The diffraction by the edges of a limited surface produces a broadening of the reflected beam pattern and a decrease of the power reflected in the specular direction. An estimation of the power reflected by a horizontally limited sheet was given by Friis et al. [1957] by using the diffraction theory for a square aperture. The 


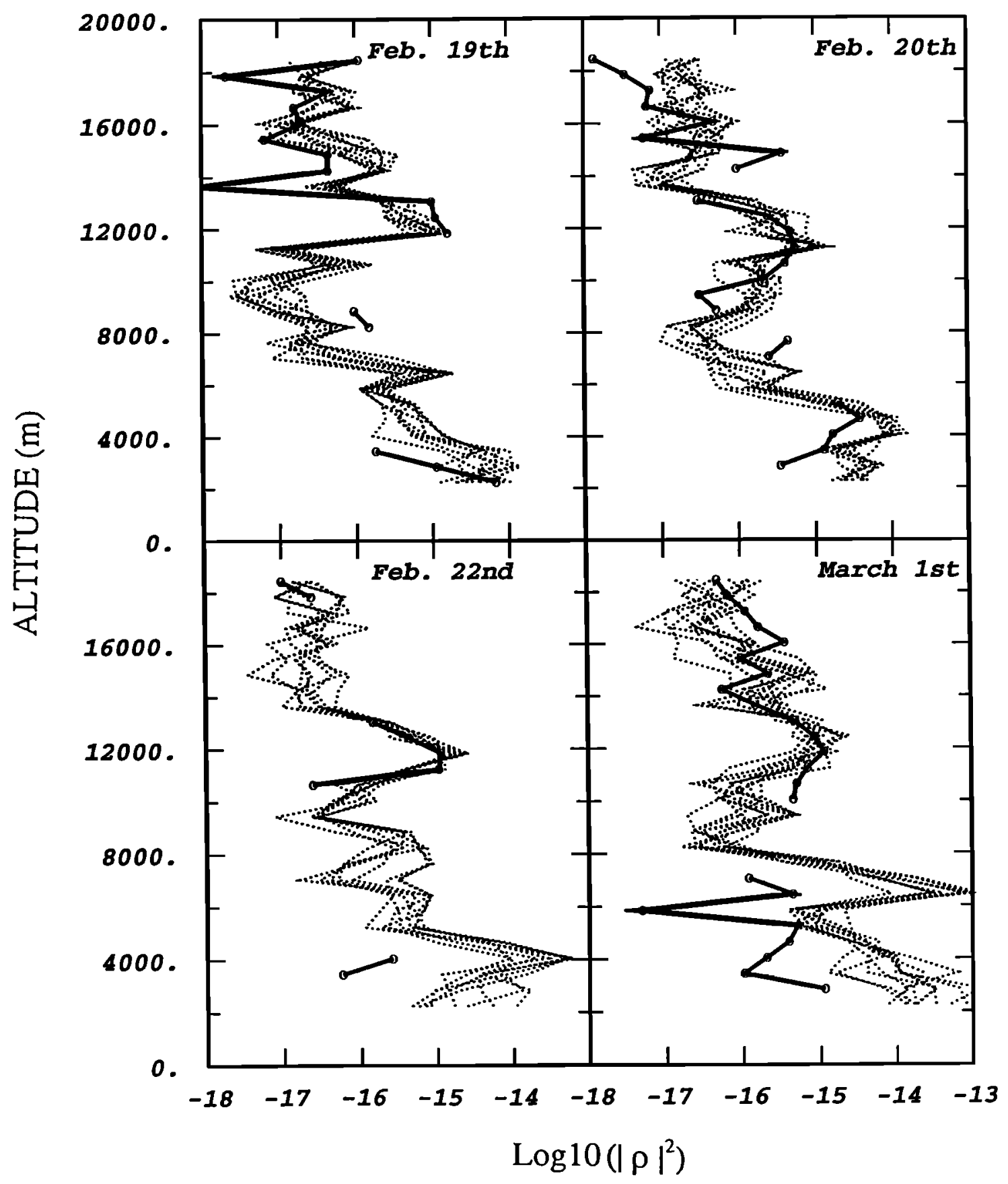

Figure 7. (a) The 45-MHz radar power reflection coefficient profiles $\log \left(|\rho|^{2}\right)$ (in dotted lines) and estimated power reflection coefficient (in $\log$ ) computed from temperature sheet set (in thick solid lines) obtained by applying the DSCV threshold (see sections 3.2 and 4.2 for more explanations). The present result is adapted from DSCV but with an altitude recalibration $(300 \mathrm{~m}$ ) and the three-sensor temperature profiles are averaged. Possible discontinuities in the reconstructed profiles are due to altitude ranges without selected sheets. It can be noted for the two sets a good agreement in shape and in level. (b) Same as in (a) but with another selected sheet set using a less drastic threshold. (c) Same as in (a) for 72.5 MHz. (d) Same as in (b) for $72.5 \mathrm{MHz}$. 


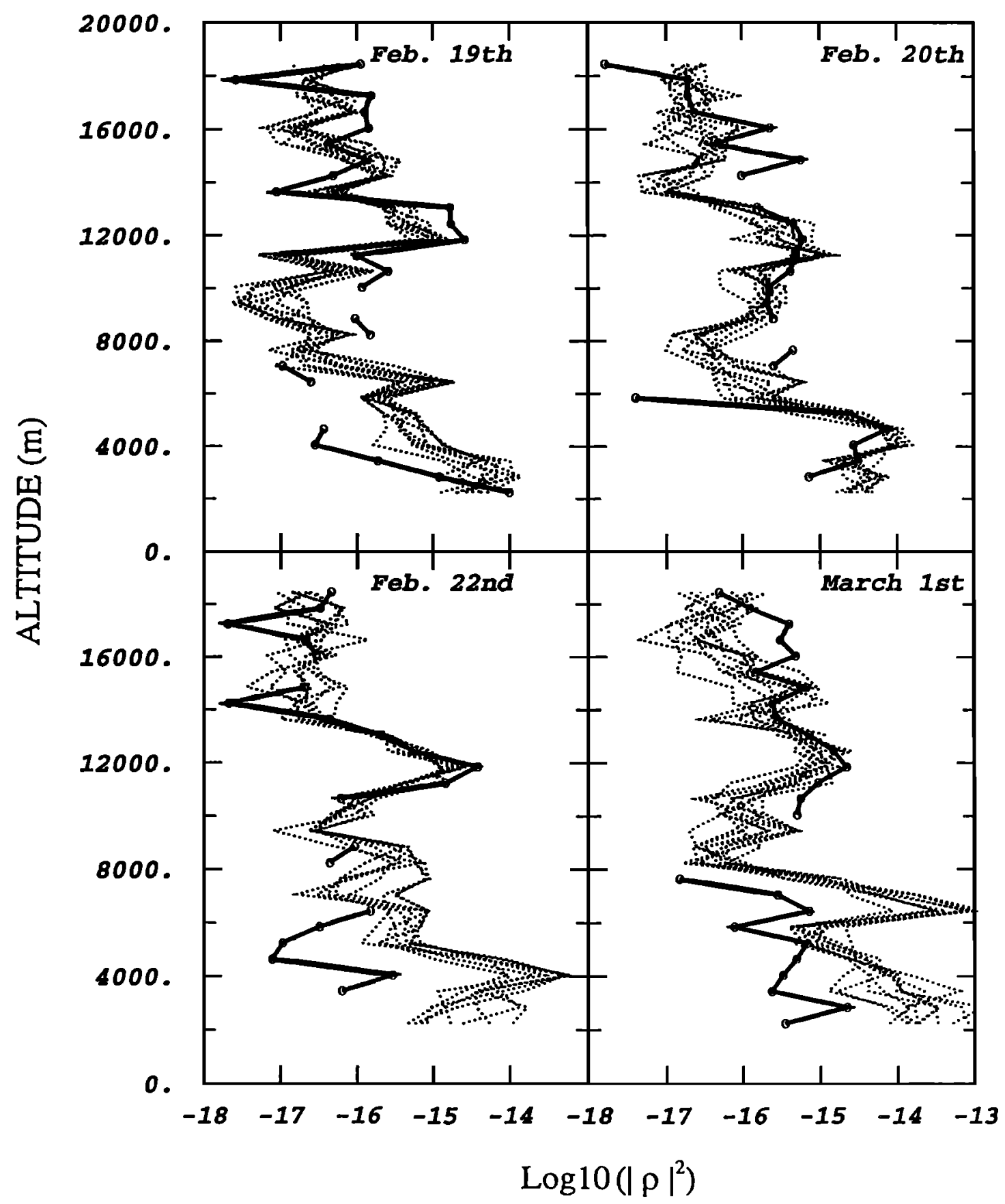

Figure 7. (continued)

sheet horizontal dimensions are supposed to be large compared with the wavelength in order to use this theory. He showed that in the Fresnel approximation, the backscattered power expression $P_{\mathrm{f}}$ (f is the finite horizontal extent) can be written:

$$
P_{f}=4 K\left\{C^{2}(u)+S^{2}(u)\right\}^{2}|\rho|_{\text {dheot }}^{2}
$$

where $|\rho|_{\text {thow }}^{2}$ is the reflection coefficient for an infinite surface, $C(u)$ and $S(u)$ are the Fresnel integrals, and $u=L /\left(\sqrt{2} R_{f}\right)$, where $L$ is the horizontal dimension of the layer. The quantities $C(u)$ can be approximated by $u$ and $S(u)$ by 0 if $u<1 / \sqrt{2}$ (or $L<R_{\mathrm{f}}$ ). Then, if $L$ is smaller than 


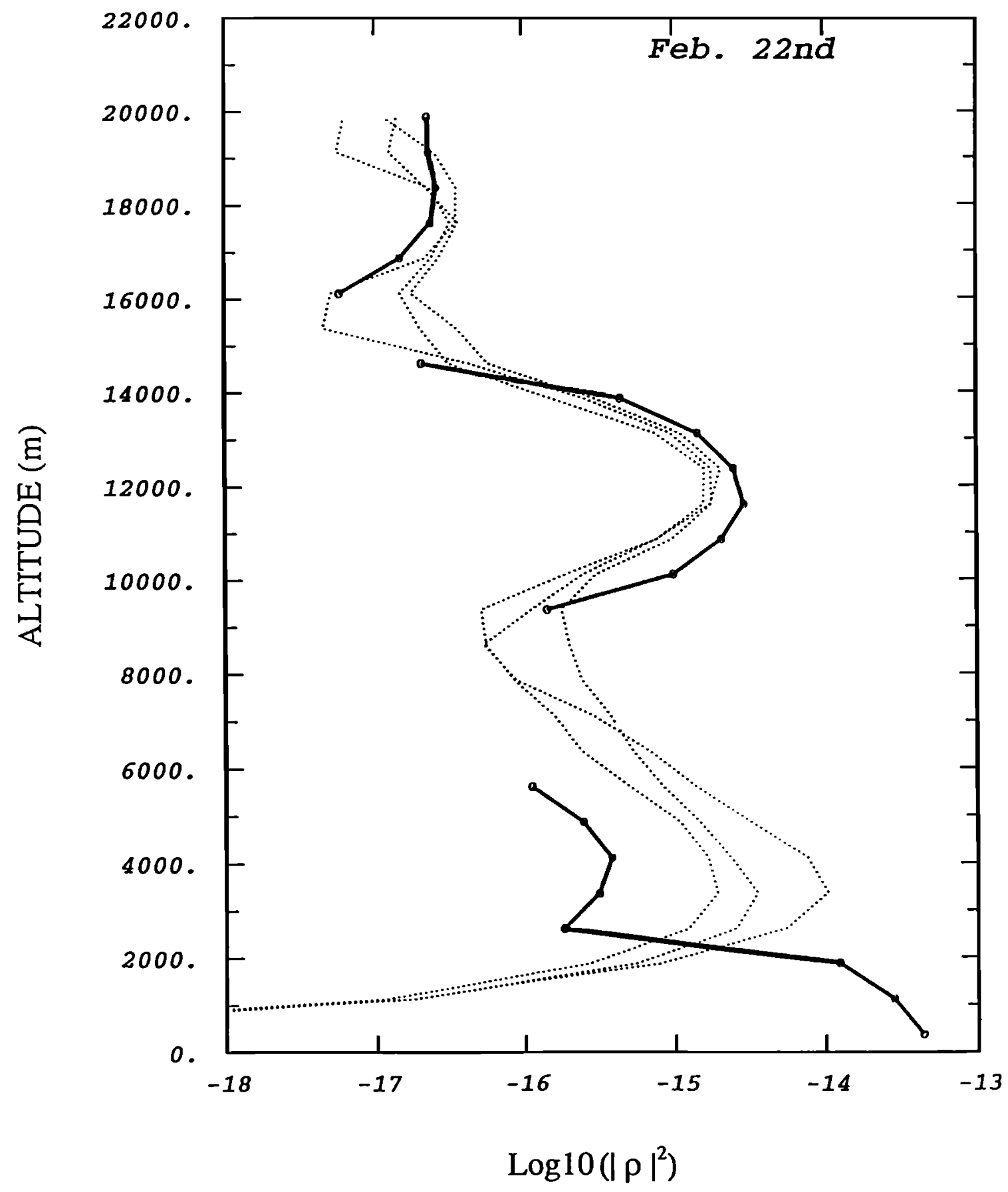

Figure 7. (continued)

the Fresnel zone radius $\boldsymbol{R}_{\mathrm{f}}$ an approximate relation can be found. The ratio between the power backscattered from a sheet of horizontal limited extent and the one backscattered from a flat and infinite sheet is then

$$
\frac{P_{f}}{P_{r}}=\left(\frac{L}{R_{f}}\right)^{4}=\frac{4 L^{4}}{\lambda^{2} r^{2}} \quad L<R_{F}
$$

This result can also be obtained from Beckmann and Spizzichino's [1963] work but only in the Fraunhofer 


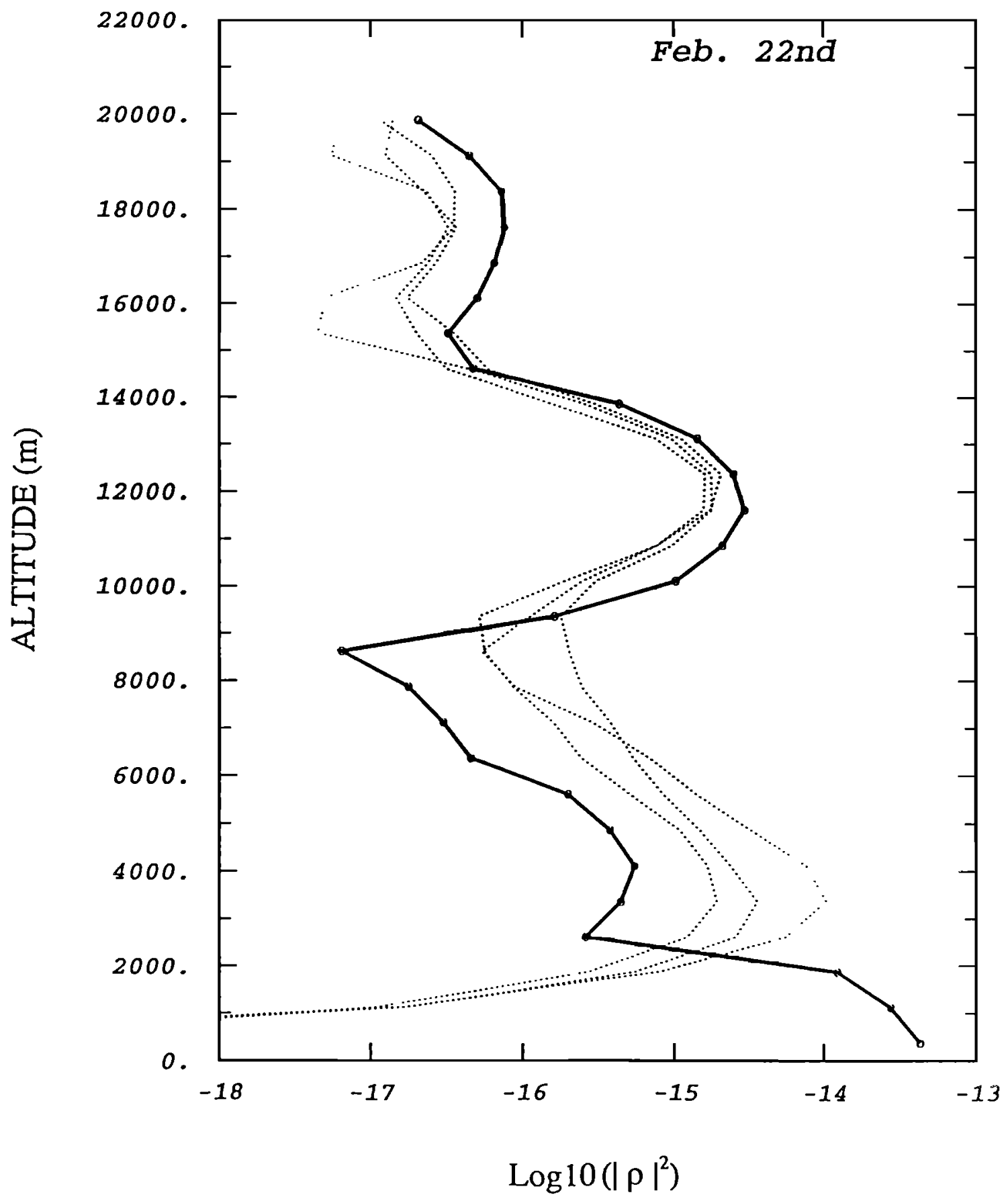

Figure 7. (continued)

zone and a similar result was introduced by Balsley and Gage [1981] by considering a circular surface instead of a square one. It can be noted that for the extreme case $L>R_{\mathrm{f}}$ that is, $u \gg 1 / \sqrt{2}, C(u)=S(u)=1 / 2$ that the classical result (4) of reflected power from an infinite area is found again. From a physical point of view the power dependence proportional to $L^{4}$ can be interpreted by a combined effect of "leakage" of the incident power outside 
the surface of the sheets and of the broadening of the diffracted power. The geometrical surface of the sheet is proportional to $L^{2}$ and the diffraction angle is proportional to $L^{-2}$, thus a combination of the two effects produce the $L^{4}$ dependence.

Power of the backscattered signal $P_{f}$ follows a $r^{4}$ range dependency law characteristic of backscatter from discrete targets. This relation allows us to give an order of loss magnitude introduced by the weighting function $\left(L / R_{f}\right)^{4}$. For example, at $10 \mathrm{~km}$ the Fresnel zone diameter is $360 \mathrm{~m}$. For a sheet of 100-m dimension, losses reach about $10 \mathrm{~dB}$. Then, to have a sufficient contribution to reflected power, horizontal sheet dimensions must be at least of the order of a Fresnel zone in length (see Table 1).

\subsection{Distortion Effects of the Sheet Surfaces}

The effect of the distortion of sheet surfaces is equivalent to focusing and defocusing effects and can produce a change of the estimated power backscattered towards the antenna. A smooth surface reflects the incident wave in specular direction while a rough one produces a broadening of the scattered beam.

As already mentioned in a previous section, little information was obtained about vertical distortions of the sheets. In particular, it is still impossible to distinguish between corrugated or random horizontal structures (DSCV).

However, in order to give an order of magnitude of losses introduced by sheet roughness, a Gaussian random assumption will be used to describe irregularity structures, keeping in mind that a Gaussian process may not be correct for this kind of structures. According to Beckmann and Spizzichino [1963], the ratio of the reflection coefficient for a rough and a flat sheet can be calculated as if the sheets were infinitely thin. The distortion model assumed by these authors is characterized by a Gaussian autocorrelation function (ACF) in the horizontal direction with a correlation length $L_{\mathrm{c}}$. Since the vertical translations do not affect the vertical $A C F$, if we assume the latter to be Gaussian, with a mean square roughness height called $h^{2}$, the statistical description of the rough sheets is equivalent to the spectral models (using oblate spheroids for the ACF) used by Doviak and Zrnic' [1984] and Hocking et al. [1990].

In the following section, slightly rough surfaces will be investigated first by using the work of Beckmann and Spizzichino [1963], who studied scattering from rough surfaces for bistatic links. Then, the facets method [e.g., Barrick, 1968], which results from physical optics and stationary phase analysis, applied to sheet surfaces will be used for the study of very rough surfaces in a follo- wing section. For both cases, strong power losses in the specular direction would disagree with the partial reflection interpretation of the aspect sensitivity.

Slightly rough surface. The Kirchhoff or physical optics method uses the "tangent plane" approximation (points of slope discontinuity are excluded) in order to obtain the approximated field on the surface. This method is valid if the following conditions are verified: $2 k r_{c} \cos \theta \gg 1$ and $1 / 2 k r_{c} \cos ^{3} \theta \gg 1$ [e.g., Valenzuela, 1978], where $r_{c}$ is the local radius of curvature of the surface and $\theta$ is the local angle of incidence (it must be noted that these conditions are not necessarily true in the real case). The scattered field in the general case of bistatic links is then obtained from the Stratton-Chu or Franz vectorial integral equations [e.g., Valenzuela, 1978]. However, Beckmann and Spizzichino [1963] used the scalar expression of the Helmotz integral by demonstrating that there is no depolarisation if the incident wave is linearly polarized for longitudinally scattered waves and thus in backscatter conditions.

Consequently, for slightly rough surfaces in the backscatter case, the conditions of application are $r_{c} \gg \lambda \pi\left(r_{c} \gg 2 \mathrm{~m}\right.$ for $45 \mathrm{MHz}$ ) in order to use the tangent plane approximation hypothesis, $4 k^{2} h^{2} \ll 1$ or $h<\lambda / 4 \pi$ ( $h<50 \mathrm{~cm}$ for $45 \mathrm{MHz}$ ) for the slightly rough approximation, and $\lambda \ll L_{c} \ll L$ for the general conditions of diffraction theory. The field is calculated in Fraunhofer zone and the multiple scattering phenomenon is neglected.

It can then be found that the ratio between the mean backscattered power and the one backscattered from a flat and infinite sheet is

$$
\begin{gathered}
\frac{\left\langle P_{r}\right\rangle}{P_{r}}=\left(\frac{L}{R_{F}}\right)^{4}\left(1+16 \pi^{3} \frac{L_{c}^{2}}{L^{2}} \frac{h^{2}}{\lambda^{2}}\right) \exp \left(-16 \pi^{2} \frac{h^{2}}{\lambda^{2}}\right) \\
\left(L<R_{F}\right)
\end{gathered}
$$

This expression includes two contributions: the specular one for $h=0$ and a diffuse one for $h \neq 0$. Since $L_{c}$ is smaller than $L, 16 \pi^{3} h^{2} \lambda^{2} L_{\mathrm{c}}^{2}$ is also small compared with $L^{2}$ and is negligible. The main factor is then $\exp \left(-16 \pi^{2} h^{2} / \lambda^{2}\right)$ with a $-4 \mathrm{~dB}$ value for $h=\lambda /(4 \pi)$ (equal to $50 \mathrm{~cm}$ at $45 \mathrm{MHz}$, limit of validity of this theory) and with a $-0.6-\mathrm{dB}$ value for $h=20 \mathrm{~cm}$ (see Table 1).

Strongly rough surface. Geometrical optics use the fact that only facets normal to the incident direction contribute to the scattered field. The surfaces must be sufficiently distorted in order that scattered fields between facets may be decorrelated and scattered powers added incoherently. The conditions of validity are (1) $k^{2} h^{2} \cos ^{2} \theta \gg 1$; for vertical incidence, this condition is 
reduced to $k^{2} h^{2}>1$ or $h>1 \mathrm{~m}$ for $6.67 \mathrm{~m}$ wavelength. (2) The tangent plane approximation must still be verified with $r_{\mathrm{s}} \gg 2 \mathrm{~m}$ at the same wavelength. Kodis [1966] introduced this concept for a perfectly conductive surface and obtained the cross section per unit area expressed as a function of the average product of the principal radii of curvature of the surface and $\langle N\rangle$ the average number of efficient facets per unit area:

$$
\sigma_{0}=\pi \overline{r_{c 1} r_{c 2}}<N>
$$

Barrick [1968] extended this result to finite conductivity and expressed the cross section per unit area in terms of the joint probability density of slopes of the surface. For a Gaussian density and for an application to sheet surfaces, this expression becomes

$$
\sigma_{0}(\theta)=\frac{1}{\cos ^{4} \theta} \operatorname{cotan}^{2}\left(\theta_{0}\right) \exp \left(-\frac{\tan ^{2} \theta}{\tan ^{2} \theta_{0}}\right)|\rho|_{\text {datat }}^{2}
$$

where $|\rho|^{2}$ is the reflection coefficient from a flat and "infinite" sheet, $\theta$ is the angle of incidence and $\tan ^{2} \theta_{0}=4 h^{2} / L_{c}^{2}$ is the mean square value of the total slope.

Then, for a vertical incidence and a horizontally isotropic pattern, the radar cross section is given by

$$
\sigma \approx \int_{\theta=0}^{\pi} \int_{\phi=0}^{2 \pi} \sigma_{0}(\theta) f^{4}(\theta) r^{2} \sin \theta \cos \theta d \theta d \phi
$$

The function $f^{2}(\theta)$ describes the power antenna beam pattern and is given approximately by

$$
f^{2}(\theta)=\exp \left(-\frac{\sin ^{2} \theta}{\sin ^{2}\left(\theta_{y_{e}}\right)}\right) \approx \exp \left(-\frac{\theta^{2}}{\theta_{y_{\varepsilon}}^{2}}\right)
$$

where $\theta_{1 / 4}^{2}=\delta \theta^{2} /(4 \ln 2)$ and $\delta \theta$ is the $3-\mathrm{dB}$ angular width $\left(5^{\circ}\right)$. After integration it can be found that

$$
\sigma \approx \pi r^{2} \frac{A^{2}}{1+A^{2}}|\rho|_{\text {abeor }}^{2}
$$

with

$$
A^{2}=\frac{8 \theta^{2}}{8 \ln 2 \tan ^{2} \theta_{0}} \approx 1.37510^{-3} \frac{L_{c}^{2}}{4 h^{2}}
$$

The mean backscattered power expressed in terms of the radar cross section is

$$
\left\langle P_{r}\right\rangle=\frac{P_{1} A_{c}^{2}}{4 \pi \lambda^{2} r^{4}} \sigma
$$

The ratio between the mean power backscattered from the rough sheet and the one backscattered from a flat and infinite sheet is

$$
\frac{\left\langle P_{r}\right\rangle}{P_{r}}=\frac{A^{2}}{1+A^{2}}=\frac{L_{c}^{2}}{L_{c}^{2}+2.910^{3} h^{2}}
$$

The ratio is equal to $-3 \mathrm{~dB}$ for $h=2.6 \mathrm{~m}$ and $L_{c}=140 \mathrm{~m}$ corresponding to a mean slope of $\theta_{0}=2.1^{\circ}$. Figure 8 shows this function for different $L_{\mathrm{c}}$ values. For $h$ values around $2.5 \mathrm{~m}$, a correlation length $L_{\mathrm{c}}$ of some $100 \mathrm{~m}$ is needed to observe a low decrease (few decibels) of the received power. An increase of $h$ and a decrease of $L_{\mathrm{c}}$ lead to a decrease of the power ratio (16) (see Table 1).

\subsection{Discussion of the Models}

It was shown that limited horizontal extent of the sheets (i.e., with a dimension less than the first Fresnel zone) can produce a large decrease of the estimated reflection coefficient. Then, the reconstructed power reflection coefficient using the simple model is realistic only if sheets have surface extents at least of the order of the Fresnel radius.

Sheet roughness was taken into account by studying the cases of slightly and very distorted surfaces. The results are only indicative and can't be taken to be representative if the required conditions of validity are not verified. The tangent plane approximation is always assumed, and a sufficient radius of curvature of surface structures with respect to the radar wavelength is needed. As expected, it can be concluded that a slightly rough surface introduces weak losses in specular direction. Distortions of a few tens of centimeters observed from high-resolution temperature profiles do not produce a substantial decrease of the estimated power reflection coefficient. In the case of a very rough surface, the power received in the specular direction strongly decreases if the correlation length of the irregularities is not sufficiently large (it has to be much larger than $100 \mathrm{~m}$ for $\mathrm{h}$ of the order of $2.5 \mathrm{~m}$ at $45 \mathrm{MHz}$ ).

\section{Discussion About Some Sheet Generation Mechanisms}

Generation mechanism(s) of these temperature sheets is/are still a very controversial subject. Several hypotheses were investigated: Bolgiano [1968] and Peltier et al. [1978] explained these temperature gradients as a consequence of Kelvin-Helmotz (KH) instabilities. The turbulence in a stable atmosphere is produced by wind shear which gives rise to $\mathrm{KH}$ instabilities. The mixing of the turbulent layer leads then to strong gradients at the boundaries. Van Zandt and Vincent [1983] suggest that these structures were associated with low-frequency buoyancy waves but Hocking et al. [1991] refuted this hypothesis because the equiphase plane of these waves 


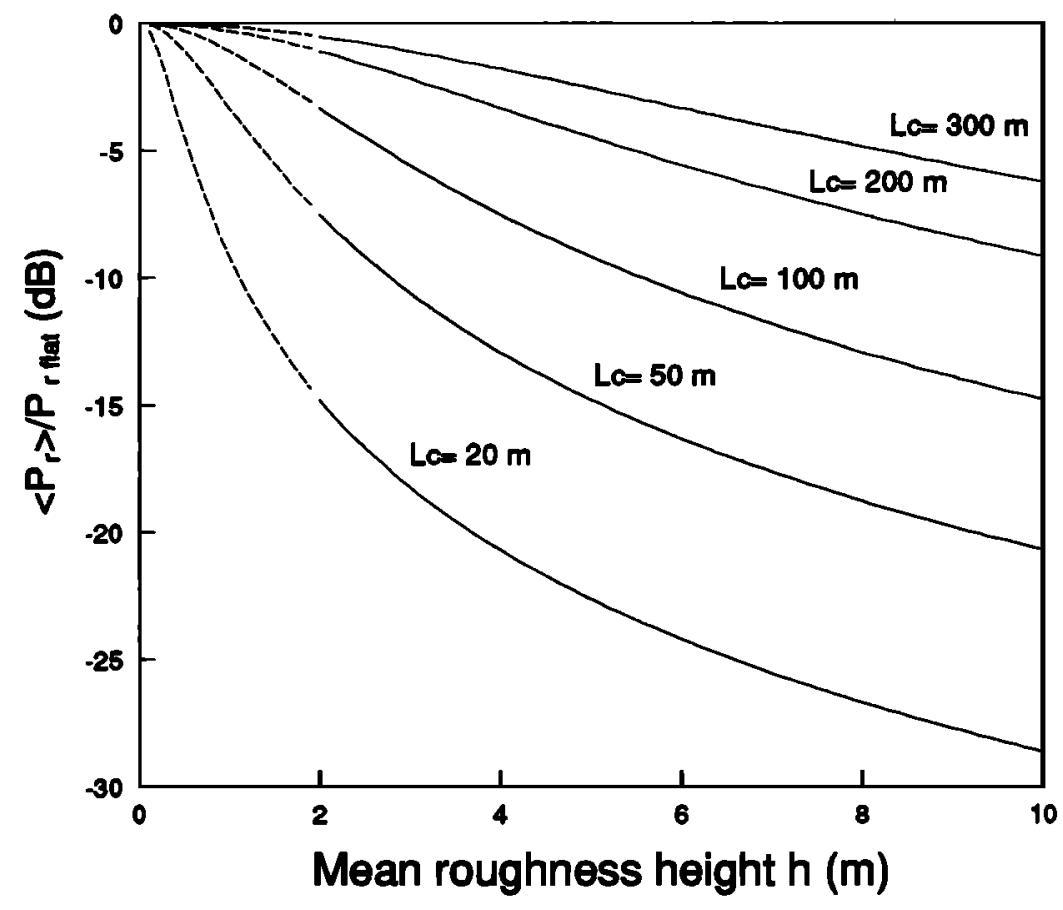

Figure 8. Attenuation effect due to distorted sheets for different correlation lengths $L_{c}$, in the case of strongly rough surfaces estimated by the facets method for a Gaussian probability density function of surface slopes. (Application conditions: $h>1 m, r_{c}>2 m$ for $45 \mathrm{MHz}$ ).

are necessarily tilted from several degrees due to presence of the viscosity. Consequently, they cannot explain the reflected echo received at vertical incidence. New possible mechanisms will not be introduced, but the aim of this section is to discuss two mechanisms which were proposed by Hocking et al. [1991] and Gossard et al. [1985].

\subsection{Viscosity and Thermal Conduction Waves:} Theoretical Hypothesis

Hocking et al. [1991] proposed that viscosity and/or thermal conduction waves could be responsible for the specular reflectors both in the stratosphere and the mesosphere. These waves would arise from partially reflected gravity waves and from gravity waves which become strongly nonlinear and then reach a critical level. They are strongly damped and they would propagate only on a vertical distance of the order of one wavelength $\lambda_{z}$. This wavelength is proportional to the square root of the viscosity (or thermal conduction) and to the temporal period $T$ of the primary gravity wave. The authors showed that the equiphase plane can be almost horizontal, justifying the vertical nature of the specular reflection. These kinds of waves would produce temperature (or density) fluctuations which, added to the mean gradient of the zone where they are created, can have the appearance of the sheets observed by our measurements. Moreover, they noted a good agreement between the vertical scales of the viscous waves and the Bragg scales of regions of highest aspect sensitivity in the stratosphere (at VHF frequencies) and in the mesosphere (at MF frequencies), supporting their hypothesis. The values of gravity waves period and viscosity parameter in the stratosphere can produce viscosity waves wavelength of the order of magnitude of the observed sheet thickness. Such agreements would then appear favorable to their theory.

Furthermore, Hocking et al. [1991] concluded that an horizontal perturbation velocity of the order of $1 \mathrm{~cm} \mathrm{~s}^{-1}$ is sufficient to generate the typical values of equivalent $C_{n}^{2}$ at $20 \mathrm{~km}$ in the stratosphere. But their quantitative result is conflicting with the $0^{\circ} 3 \mathrm{~K}$ temperature increase for a 3-m wavelength $\lambda_{z}$ of the viscous wave, because 1 $\mathrm{m} \mathrm{s}^{-1}$ wind perturbation is needed in this case (bottom right, page 1291 of their paper). This (small) computed value of velocity gradient is incorrect due to an error in the expression of refractive index (formula (20) of their paper) where the pressure $p$ is assumed to be in Pascals, while it should be taken in millibars with the given numerical coefficient. This error leads to an underesti- 
Table 1. Orders of magnitude of losses

\begin{tabular}{|c|c|c|c|c|c|c|c|c|}
\hline \multirow{3}{*}{$\begin{array}{c}r, m \\
5000 \\
10000 \\
15000\end{array}$} & \multicolumn{2}{|c|}{$\begin{array}{c}\boldsymbol{R}_{\boldsymbol{f}} \\
\text { Fresnel Radius, m }\end{array}$} & \multicolumn{6}{|c|}{$\begin{array}{l}\text { Limited Extent Effects } \\
\qquad P_{f} P_{r}=\left(L / R_{f}\right)^{4}, \mathrm{~dB}\end{array}$} \\
\hline & \multirow{2}{*}{$\begin{array}{c}45 \mathrm{MHz} \\
129 \\
182 \\
223\end{array}$} & \multirow{2}{*}{$\begin{array}{c}72.5 \mathrm{MHz} \\
102 \\
144 \\
176\end{array}$} & \multicolumn{2}{|c|}{$L=50 \mathrm{~m}$} & \multicolumn{2}{|c|}{$L=100 \mathrm{~m}$} & \multicolumn{2}{|c|}{$L=150 \mathrm{~m}$} \\
\hline & & & $\begin{array}{l}-16.5 \\
-22.4 \\
-26.0\end{array}$ & $\begin{array}{l}-12.3 \\
-18.4 \\
-21.8\end{array}$ & $\begin{array}{l}-4.4 \\
-10.4 \\
-13.9\end{array}$ & $\begin{array}{l}-0.3 \\
-6.3 \\
-9.8\end{array}$ & $\begin{array}{l}0 \\
-3.3 \\
-6.8\end{array}$ & $\begin{array}{c}0 \\
0 \\
-2.7\end{array}$ \\
\hline \multirow[b]{2}{*}{$h, \mathrm{~m}$} & \multicolumn{8}{|c|}{$\begin{array}{l}\text { Slightly Rough Surface Effects } \\
\left\langle P_{r}>P_{r} \approx \exp \left(-16 \pi^{2} h^{2} / \lambda^{2}\right), \mathrm{dB}\right.\end{array}$} \\
\hline & \multicolumn{4}{|c|}{$45 \mathrm{MHz}$} & \multicolumn{4}{|c|}{$72.5 \mathrm{MHz}$} \\
\hline $\begin{array}{l}0.1 \\
0.2 \\
0.5\end{array}$ & \multicolumn{4}{|c|}{$\begin{array}{l}-0.1 \\
-0.6 \\
-3.9\end{array}$} & \multicolumn{4}{|c|}{$\begin{array}{c}-0.4 \\
-1.6 \\
\text { not applicable }\end{array}$} \\
\hline
\end{tabular}

\begin{tabular}{ccccc} 
& \multicolumn{5}{c}{$\begin{array}{l}\text { Strongly Rough Sufface Effects } \\
\left\langle P_{r}>>P_{r}=L_{c}^{2}\left(L_{c}^{2}+2.910^{3} h^{2}\right), \mathrm{dB}\right.\end{array}$} \\
\cline { 2 - 5 }$h, \mathrm{~m}$ & $L_{c}=50 \mathrm{~m}$ & $L_{\mathrm{c}}=100 \mathrm{~m}$ & $L_{\mathrm{c}}=200 \mathrm{~m}$ & $L_{\mathrm{c}}=300 \mathrm{~m}$ \\
\hline 2.5 & -9.2 & -4.5 & -1.6 & -0.8 \\
4 & -12.9 & -7.5 & -3.3 & -1.8 \\
6 & -16.3 & -10.6 & -5.5 & -3.3 \\
\hline
\end{tabular}

Orders of magnitude of losses produced by the limited horizontal extent of the sheets and by their possible distorted surfaces for the "slightly rough" and the "strongly rough" cases using Gaussian statistic treated in the text for $45 \mathrm{MHz}$ and $72.5 \mathrm{MHz}$. The parameter ${ }_{z}$ is the distance sheet radar, $h$ is the mean roughness height, and $L_{c}$ is the correlation length of the irregularities. These results show that horizontal sheet dimensions must be at least of the onder of a Fresnel zone in length, and $h$ must be less than about $20 \mathrm{~cm}$ for the slightly rough case in order to have weak losses. In case of greater roughness height (several meters), $L_{\varepsilon}$ must be of the order of several hundreds of meters in order to have losses of the same order (less than $3 \mathrm{~dB}$ ).

mate of the velocity perturbation by a factor of 100 ; the authors should have then obtained an order of $1 \mathrm{~m} \mathrm{~s}^{-1}$ instead of $1 \mathrm{~cm} \mathrm{~s}^{-1}$. Consequently, the viscosity waves model in its present form does not seem adequate because it would predict unstable sheets associated with unrealistic wind perturbations. However, the physical model behind viscosity waves seems interesting and it is possible that a modified version of this model can account for some of the observed sheets.

\subsection{Planetary Boundary Layer Observations and "Sheet and Layer" Models}

Gossard et al. [1985] presented a "sheet and layer" model where the generation of such structures is asso- ciated with turbulence. The authors pointed out the presence of stable temperature sheets associated with fine humidity and wind structures under $300 \mathrm{~m}$ height in the boundary layer. In the present paper the connection between temperature and humidity structures (in the troposphere) was already discussed in section 3.2 . In the boundary layer these sheets are generally preceded by large positive heat fluxes associated with superadiabatic zones resulting from a breaking gravity wave phenomenon. Such superadiabatic zones are not observed from our detailed temperature measurements in the troposphere and stratosphere. But, the idea that the temperature sheets can be associated with turbulent layers is partially supported by observations in DSCV where the presence of turbulence just above or below 
some of the sheets was already noted. However, no systematic investigation was performed and a definite conclusion will be reached only when all the available data, including velocity, are carefully analyzed.

\section{Conclusion}

Colocated VHF ST radar and balloon measurements were simultaneously performed during the RASCIBA campaign in February and March 1990. Examinations of the radar echo profiles show aspect sensitivity (vertical echo enhancement with regard to the oblique ones) at the $6.67 \mathrm{~m}$ wavelength (45-MHz frequency) as it could be expected from previous experiments at similar wavelength [e.g., Gage and Green, 1978; Sato et al., 1985]. But it was shown for the first time that an overhead enhancement can also be very large (up to about $15 \mathrm{~dB}$ ) at the $4-\mathrm{m}$ wavelength $(72.5-\mathrm{MHz}$ frequency).

The refractive index structures responsible for the aspect sensitivity were not previously clearly identified. This was mainly due to the lack of in situ observations with a sufficient resolution. However, thin layer structures were assumed by several investigators [e.g., Röttger and Liu, 1978] to be at the origin of the aspect sensitivity but have not previously been observed with sufficient resolution. The presented in situ experiments provide vertical temperature profiles at very high resolution $(20 \mathrm{~cm})$ that reveal the existence of these structures as temperature "sheets." Enhancement of the radar signal in the vertical direction is clearly related to the occurrence and location of these gradients (Figure 6). This fact indicates moreover that the horizontal extent of the altitude zones favourable to sheet presence is longer than several tens of kilometers corresponding to the distance of the balloons from the radar site (reaching 130 kilometers for one of the four balloon flights). In light of these temperature sheet measurements the partial reflection mechanism was then investigated to explain the VHF aspect sensitivity by using a simple model that assumes a flat and largely extended sheet. Reconstructed power reflection coefficient profiles were compared with those obtained from radar soundings. The two kinds of profiles (Figure 7) are quite similar in strength without any normalization factor and in shape, suggesting that partial reflection from temperature sheets is an important and generally dominant process in the vertical direction. But the lack of information about horizontal extent and surface roughness prevented the computation of an accurate power reflection coefficient profile. The strong correspondence of computed and observed $|\rho|^{2}$ suggests that these effects are small. However, under some assumptions, such as sheet horizontal extent greater than the Fresnel zone and very slightly rough surface, the computed values can stay in the same order of magnitude. In case of strongly rough surfaces ( $h$ of the order of a few meters), the correlation length of the irregularities must be larger than hundreds of meters in order to have weak losses in the specular direction (i.e., very small slopes are needed). The angular variations of the echo power observed by several authors [e.g. Tsuda et al., 1988; Chu et al., 1990] can be explained by sheet roughness [Röttger, 1980a] although a more detailed study is necessary to confirm this hypothesis.

The well known correlation between the vertical radar power and $N^{4}$ [e.g., Tsuda et al., 1988; Dalaudier et al., 1989] is compatible with the partial reflection hypothesis from sheets because they are generally located in regions of high stability. However, the vertical sheet distribution does not allow to account quantitatively for the exponent 4. This observation does not explain by itself the reason of their existence and what sort of mechanism(s) generate(s) these structures. Two families of mechanisms were proposed to explain the origin of the sheets: generation mechanisms associated either with waves or with turbulence. The direct association of sheets with gravity waves was ruled out by Hocking et al. [1991] because of the necessary inclusion of viscosity in the equations. However, the alternative model proposed by these authors, viscosity waves, was shown to be inadequate in its present form because of a large error in a numerical coefficient. Nevertheless, the viscosity waves interpretation is not completely excluded for some of the observed sheets. On the other hand, the sheet and layer model, used by Gossard et al. [1985] in the boundary layer, could be adapted for higher altitude and then account for the part of observed sheets that are associated with turbulent layers. More definitive conclusions on this subject are expected from further investigations, currently performed, on the global present data set.

Future experimental investigations are thus needed for a better understanding of the 3-D temperature field. A better knowledge of the morphology of the fine scale refractive index structures will allow a complete understanding of the aspect sensitivity at VHF frequencies. A 2-D sounding of the temperature sheets would improve the knowledge about their horizontal structures and would lead to a global understanding of the partial reflection contribution at vertical incidence. Such a description would be very useful to get an insight on the generation, evolution, and destruction mechanisms of the sheets which are presently largely unknown. In a parallel way a better knowledge of the vertical enhancement mechanism can be obtained by an improvement of the radar system. A better vertical resolution (better than $600 \mathrm{~m}$ ) with a narrower antenna beam and 
the introduction of interferometric methods can be very adequate to observe the aspect sensitivity in light of future atmospheric sheet observations.

Acknowledgments. We thankJ. Vernin and M. Azouit from the University of Nice for their cooperation, C. Bourdier, J. Gagelli and Y. Ruin from the LSEET for ST radar implementations on the site; B. Lamy, J. L. Caccia, and E. Spano for experimental assistance, and S. Ferrat for making available error estimations program. We thank J. C. Génie, Y. Nizou, M. Leclère, and P. Hulin from the Service d'AĆronomie and the ST network national group to have made available the INSU METEO $72.5 \mathrm{MHz}$ radar. We thank also all the balloon team from the CNES (Centre National d'Etudes Spatiales) and M. Faucon and Vincent from the CNES for allowing the two ST radar implementations on the launching site and making all facilities available to the radar team. This program was supported by PAMOY (Programme Atmosphere Moyenne) and RADAR ST OPERATION from INSU-CNRS (Institut National des Sciences de l'UniversCentre National de la Recherche Scientifique). Constructive comments from reviewers are greatly appreciated and helped to improve the final manuscript.

\section{References}

Balsley, B. B., and K. S. Gage, On the vertical-incidence VHF backscattered power profile from the stratosphere, Geophys. Res. Lett., 8, 1173-1175, 1981.

Balsley, B. B., and V. L. Peterson, Doppler-radar measurements of clear air atmospheric turbulence at $1290 \mathrm{MHz}, J$. Appl. Meteorol., 20, 266-274, 1981.

Barrick, D. E., Rough surface scattering based on the specular point theory, IEEE Trans. Antennas Propag., 16, 449-453, 1968.

Beckmann, P., and A. Spizzichino, The Scattering of Electromagnetic Waves From Rough Surfaces, Pergamon, Tarrytown, N. Y., 1963.

Bolgiano, R., Jr., The general theory of turbulence: Turbulence in the atmosphere, in Wind and Turbulence in the Stratosphere, Mesosphere and Ionosphere, edited by $\mathrm{K}$. Rawer, p. 371, North-Holland, New York, 1968.

Booker, H. G, and W. E. Gordon, A theory of radio scattering in the troposphere, Proc. IEEE, 38, 401-412, 1950.

Chu, Y. H., J. K. Chao, C. H. Liu, and J. Röttger, Aspect sensitivity at tropospheric heights measured with vertically pointed beam of the Chung-Li VHF radar, Radio Sci., 25, 539-550, 1990.

Dalaudier, F., M. Crochet, and C. Sidi, Direct comparison between in situ and radar measurements of temperature fluctuation spectra: A puzzling result, Radio Sci., 24, 311-324, 1989.

Dalaudier F., C. Sidi, M. Crochet, and J. Vernin, Direct evidence of "sheets" in the atmospheric temperature field, J. Atmos. Sci., 51, 237-248, 1994.

Doviak, R. J., and D. S. Zmic', Reflection and scatter formula for anisotropically turbulent air, Radio Sci., 19, 325-336, 1984. du Castel F., Tropospheric Radio Wave Propagation Beyond the Horizon, Pergamon, Tarrytown, N. Y., 1966.

Friend, A. W., Theory and practice of tropospheric sounding by radar, Proc. IEEE, 37, 116-138, 1949.

Friis, H. T., A. B. Crawford and D. C. Hogg, A reflection theory for propagation beyond the horizon, Bell Sys. Tech. $J ., 36,627-644,1957$.

Gage, K. S., and B. B. Balsley, On the scattering and reflection mechanisms contributing to clear air radar echoes from the troposphere, stratosphere, and mesosphere, Radio Sci., 15, 243-257, 1980.

Gage, K. S., and J. L. Green, Evidence for specular reflection from monostatic VHF radar observations of the stratosphere, Radio Sci., 13, 991-1001, 1978.

Gage, K. S., B. B. Balsley, and J. L. Green, Fresnel scattering model for the specular echoes observed by VHF radar, Radio Sci., 16, 1447-1453, 1981.

Gage K. S., W. L. Ecklund, and B. B. Balsley, A modified Fresnel scattering model for the parametrization of Fresnel returns, Radio Sci., 20, 1493-1501, 1985.

Gossard, E. E., J. E. Gaynor, R. J. Zamora, and W. D. Neff, Fine structure of elevated stable layers observed by sounder and in situ tower sensors, J. Atmos. Sci., 42, 2156-2169, 1985.

Green, J. L., and K. S. Gage, Observations of stable layers in the troposphere and stratosphere using VHF radar, Radio Sci., 15, 395-405, 1980.

Green, J. L., K. S. Gage, T. E. Van Zandt, Atmospheric measurements by VHF pulsed Doppler radar, IEEE Trans. Geosci. Electron., 17, 262-280, 1979.

Gurvich, A. S., and A. I. Kon, Aspect sensitivity of radar returns from anisotropic turbulent irregularities, J. Electromagn. Waves Appl., 7, 1343-1353, 1993.

Hocking, W. K., Measurements of turbulent energy dissipation rates in the middle atmosphere by radar techniques: A review, Radio Sci., 20, 1403-1422, 1985.

Hocking, W. K., and J. Röttger, Pulse length dependence of radar signal strengths for Fresnel backscatter, Radio Sci., 18, 1312-1324, 1983.

Hocking, W. K., S. Fukso, T. Tsuda, M. Yamamoto, T. Sato, and S. Kato, Aspect sensitivity of stratospheric VHF radio wave scatterers, particularly above $15-\mathrm{km}$ altitude, Radio Sci., 25, 613-627, 1990.

Hocking, W. K., S. Fukao, M. Yamamoto, T. Tsuda, and S. Kato, Viscosity waves and thermal-conduction waves as a cause of "specular" reflectors in radar studies of the atmosphere, Radio Sci., 26, 1281-1303, 1991.

Imberger, J., and G. N. Ivey, On the nature of turbulence in stratified fluid, II, Applications to lakes, J. Phys., Oceanogr., 21, 659-680, 1991.

Kodis, R. D., A note on the theory of scattering from an irregular surface, IEEE Trans. Antennas Propag, 14, 77-82, 1966.

Luek, R. G., Turbulent mixing at the Pacific subtropicalfront, J.Phys. Oceanogr., 18, 1761-1774, 1988.

Lumley, J. L., The spectrum of nearly inertial subrange in a stably stratified fluid, J. Atmos. Sci., 21, 99-102, 1964. 
Misme, P., F. du Castel and J. Voge, Reflexions partielles dans l'atmosphère et propagation à grande distance. Première partie: Mesures météorologiques, Ann. Télécommun., 13, 209-214, 1958.

Ottersten, H., Mean vertical gradient of potentiel refractive index in turbulent mixing and radar detection of C.A.T., Radio Sci., 4, 1247-1249, 1969a.

Ottersten, H., Radar backscattering from the turbulent clear atmosphere, Radio Sci., 4, 1251-1255, $1969 \mathrm{~b}$.

Peltier, W.R., J. Halle, and T.L. Clark, The evolution of finite amplitude Kelvin-Helmhotz billows, Geophys. Astrophys. Fluid Dyn., 10, 53-87, 1978.

Ratcliffe, J.A., Some aspects of diffraction theory and their application to the ionosphere, Rep. Prog. Phys., 19, 188-267, 1956.

Röttger, J., Reflection and scattering of VHF radar signals from atmospheric refractivity structures, Radio Sci., 15, 259-276, 1980a.

Röttger, J., Structure and dynamics of the stratosphere and the mesosphere revealed by VHF radar investigations, Pure Appl. Geophys., 118, 494-527, 1980 b.

Rö̈tger, J., and C. H. Liu, Partial reflection and scattering of VHF radar signals from the clear atmosphere, Geophys. Res. Lett., 5, 357-360, 1978.

Sato, T., and R. F. Woodman, Fine altitude resolution observation of stratospheric turbulent layers by the Arecibo $430 \mathrm{MHz}$ radar, J. Atmos. Sci., 39, 2546-2552, 1982.

Sato, T., T. Tsuda, S. Kato, S. Morimoto, S. Fukao, and I. Kimura, High-resolution MST observations of turbulence by using the MU radar, Radio Sci., 20, 1452-1460, 1985.

Saxton, J. A., J. A. Lane, R. W. Meadows, and P. A. Matthews, Layer structure of the troposphere, Proc. Inst. Electr. Eng., 111, 275-283, 1964.

Sheen, D. R., C. H. Liu, and J. Röttger, A study of signal statistics of VHF radar echoes from clear air, J. Atmos. Terr. Phys., 47, 675-684, 1985.

Shur, G. H., Experimental studies of the energy spectrum of atmmsopheric turbulence, Proc. Cent. Aerol. Obs. U.S.S.R., 15, 79-90, 1962.

Tatarski, V. I., Wave Propagation in a Turbulent Medium, translated by R. A. Silverman, McGraw-Hill,New York, 1961.
Tsuda, T., T. Sato, K. Hirose, S. Fukao, and S. Kato, MU radar observations of the aspect sensitivity of backscattered VHF echo power in the troposphere and the lower stratosphere, Radio Sci., 21, 971-980, 1986.

Tsuda, T., P. T. May, T. Sato, S. Kato, and S. Fukao, Simultaneous observations of reflection echoes and refractive index gradient in the troposphere and lower stratosphere, Radio Sci., 23, 655-665, 1988.

Valenzuela, G. R., Scattering of electromagnetic waves from the ocean, in Surveillance of Environmental Pollution and Resources by Electromagnetic Waves, Edited by T. Lund, pp. 199-226, D. Reidel Publishing Compagny, London, 1978.

Van Zandt, T. E., and R. A. Vincent, Is VHF Fresnel reflectivity due to low frequency buoyancy waves?, in Handbook for MAP, Vol. 9, pp. 78-80, SCOSTEP Secr., Univ. of IIl., Urbana, Ill., 1983.

Vernin J., M. Crochet, M. Azouit, and O. Ghebrebrhan, SCIDAR/radar simultaneous measurements of atmospheric turbulence, Radio Sci., 25, 953-959, 1990

Wait,J.R.,Electromagnetic Waves in Stratified Media, chap. IV, 2nd ed., Pergamon, Tarrytown, N. Y., 1970.

Waterman, A. T., T. Z. Hu, P. Czechowsky, and J. Röttger, Measurement of anisotropic permittivity structure of upper troposphere with clear air radar, Radio Sci., 20, 1580-1592, 1985.

Weinstock, J., Vertical turbulent diffusion in a stably stratified fluid, J. Atmos. Sci., 35, 1022-1027, 1978.

Woodman, R. F., and Y. H. Chu, Aspect sensitivity measurements of VHF backscatter made with the Chung-Li radar: Plausible mechanisms, Radio Sci., 24, 113-125, 1989.

M. Crochet and H. Luce LSEET, Université de Toulon et du Var, CNRS UA 705, B.P. 132, 83957, La Garde, France. (e-mail: luce@lseet.univ-tlnfr)

F.Dalaudier and C. Sidi, Service d'Aéronomie du CNRS, Verrières le Buisson, France (e-mail: dalaudie@savtcp.aerov.jussieu.fr)

(Received April 30, 1994; revised September 30, 1994; accepted November 30,1994) 